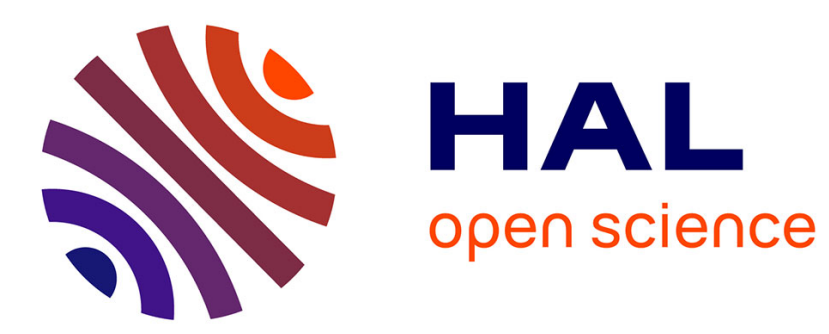

\title{
Averaged rotational dynamics of an asteroid in tumbling rotation under the YORP torque
}

\author{
S. Cicalò, D. J. Scheeres
}

\section{To cite this version:}

S. Cicalò, D. J. Scheeres. Averaged rotational dynamics of an asteroid in tumbling rotation under the YORP torque. Celestial Mechanics and Dynamical Astronomy, 2010, 106 (4), pp.301-337. 10.1007/s10569-009-9249-7 . hal-00568372

\section{HAL Id: hal-00568372 \\ https://hal.science/hal-00568372}

Submitted on 23 Feb 2011

HAL is a multi-disciplinary open access archive for the deposit and dissemination of scientific research documents, whether they are published or not. The documents may come from teaching and research institutions in France or abroad, or from public or private research centers.
L'archive ouverte pluridisciplinaire HAL, est destinée au dépôt et à la diffusion de documents scientifiques de niveau recherche, publiés ou non, émanant des établissements d'enseignement et de recherche français ou étrangers, des laboratoires publics ou privés. 


\title{
Averaged rotational dynamics of an asteroid in tumbling rotation under the YORP torque
}

\author{
S. CICALÒ ${ }^{1}$ and D. J. SCHEERES ${ }^{2}$ \\ ${ }^{1}$ Department of Mathematics, University of Pisa \\ Largo B. Pontecorvo 5, 56127 Pisa, Italy \\ ${ }^{2}$ Department of Aerospace Engineering Sciences \\ Colorado Center for Astrodynamics Research \\ The University of Colorado at Boulder \\ 429 UCB Boulder, CO 80309-0429 \\ e-mailscicalo@mail.dm.unipi.it; scheeres@colorado.edu
}

\begin{abstract}
The secular effect of YORP torque on the rotational dynamics of an asteroid in non-principal axis rotation is studied. The general rotational equations of motion are derived and approximated with an illumination function expanded up to second order. The resulting equations of motion can be averaged over the fast rotation angles to yield secular equations for the angular momentum, dynamic inertia and obliquity. We study the properties of these secular equations and compare results to previous research. Finally, an application to several real asteroid shapes is made, in particular we study the predicted rotational dynamics of the asteroid Toutatis, which is known to be in a non-principal axis state.
\end{abstract}

Key words: asteroid, rotational dynamics, YORP effect, tumbling rotation.

\section{Introduction}

The aim of this work is to study the rotational dynamics of a celestial body under the YORP torque. The YORP (Yarkovsky-O'Keefe-RadzievskiiPaddack) torque is the consequent effect to infrared thermal emission from the surface of a body irradiated by the Sun, and was first introduced in [Rubincam 2000]. This effect is mainly important for the rotational dynamics of small solar system bodies, particularly asteroids of size $<10 \mathrm{~km}$. Specifically, it can have a strong influence on the evolution of the obliquity 
and of the rotation rate of these bodies, and it may have also a role in the creation of binary asteroids.

The dynamics of the YORP effect has been previously studied by many authors and in different ways. With numerical models by

[Capek \& Vokrouhlicky 2004] and [Vokrouhlicky \& Capek 2002].

An analytical description of the effect is given, for example, in

[Nesvorny \& Vokrouhlicky 2007], in [Scheeres \& Mirrahimi 2008] and more recently in [Mysen 2008]. As the YORP torque is a small torque, it can be treated as a perturbation to the unperturbed rotational motion, and the important effects are then secular ones over long time spans. Thus the analytical solutions given in these papers are basically first order, in the small quantity YORP torque over angular momentum, averaged solutions over the rotational and the orbital motion of the asteroid, and they are based on the hypothesis of principal axis rotation around the maximum moment of inertia.

However, there are examples of asteroids which are not in principal axis rotation, said to be in tumbling rotation, and in such cases the previous analytical models cannot be applied (see [Hudson \& Ostro 1995]). The first work dedicated to the study of these rotators was [Vokrouhlicky et al. 2007], in which a full numerical integration of the equations of motion is performed. In this work we derive a first analytical theory that can explain the secular evolution behaviour of a non-principal axis rotator.

In [Neishtadt et al. 2003] and [Sidorenko et al. 2008] the generic evolutionary equations for an outgassing comet nucleus rotation were developed. Here we will follow a similar approach, looking for the averaged first order equations of a generic rigid body under the thermal YORP torques, without the hypothesis of principal axis rotation.

In addition to the so called "Rubincam's approximation", which consists of assuming zero thermal conductivity for the surface of the body, there are some others limitations in our analytical approach, in particular in modelling the illuminated-shaded portion of the body's surface hit by the Sun. We should continue to use general, non-convex bodies in our studies but note that we make the strong approximation that there is no self-shadowing i.e., model them as locally convex. Thus this work does not pretend to be a complete analytical theory of tumbling rotators under YORP, but is a first step in this direction.

The solution of the evolutionary equations that we find turns out to be easily describable in terms of few suitable quantities. The results and the conclusions that we find here include and are in agreement with the previous analytical results about principal axis rotators, but they are only partially in agreement with the numerical results in [Vokrouhlicky et al. 2007], although they have in common all of the main features seen in that work. Better ap- 
proximations are needed and we briefly discuss some possible generalizations of the theory in a qualitative way.

In section 2 we describe the full differential equations of the rotational dynamics of a rigid body under a torque, and we define the fundamental reference systems. We describe the equations for the YORP torque and for the energy dissipation dynamics. Under the assumption of no resonance between the rotation of the body and its orbital motion, in section 3 we obtain the first order (in the small parameter YORP torque over angular momentum) averaged equations for the angular momentum vector $\mathbf{L}$ of the asteroid and for the so called dynamic inertia $I_{D}$, which is a parameter related to the non-principal rotation mode of the body. In section 4 we discuss the dynamics without energy dissipation for the angular momentum vector and the dynamic inertia along with some important generalizations. In section 5 we describe the chaotic behaviour of the rotational dynamics when the body is close to the critical solution $I_{D}=B$, where $B$ is the intermediate moment of inertia. Finally in section 6 we apply our analytical results to some real asteroid shape models.

\section{Rotational dynamics}

Our dynamical problem is the rotation of a rigid body under a torque. In order to write the differential equations that describe this dynamics, we have to define some suitable reference systems.

\section{$2.1 \quad$ Reference systems}

The first important reference system is the body fixed, principal axis of inertia system. We indicate with $\xi, \eta, \zeta$ the coordinates relative to the principal inertia axes. In particular $\xi$ is relative to the minimum moment $A, \eta$ to the intermediate moment $B$, and $\zeta$ to the maximum moment of inertia $C$.

The second system that we define is the inertial, orbital reference system $X, Y, Z$. Where $\mathbf{Y}$ is normal to the orbit and $\mathbf{Z}$ is along the perihelion direction.

The third system is the angular momentum reference system, that we indicate with $x, y, z$. Where $\mathbf{z}$ is along the angular momentum vector direction $(\mathbf{L}=L \mathbf{z})$, and the axis $\mathbf{y}$ lies in the plane $(\mathbf{X}, \mathbf{Y})$.

\section{$2.2 \quad$ Angular variables}

The previous reference systems can be related to each other by suitable rotations, and the coordinates by suitable rotation matrices. To move the 

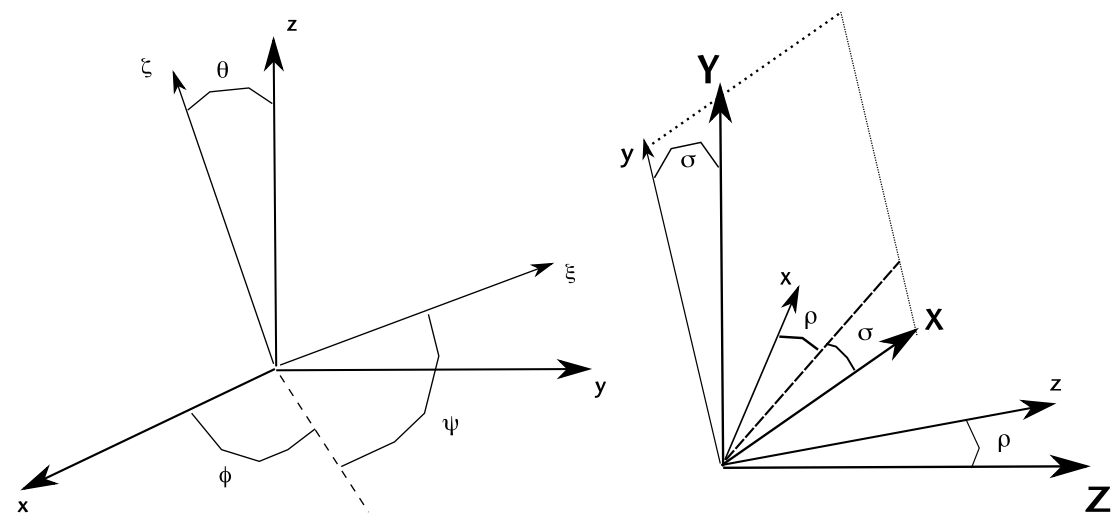

Figure 1: On the left the angular momentum reference system $x, y, z$ and the body fixed reference system $\xi, \eta, \zeta$, related by the Euler angles $\phi, \theta, \psi$. On the right the orbital reference system $X, Y, Z$ and the angular momentum reference system related by the rotation angles $\rho, \sigma$.

$x, y, z$ reference system to the $\xi, \eta, \zeta$, we need one rotation of $\phi$ around the third axis, one rotation of $\theta$ around the first axis and one rotation of $\psi$ around the third axis. To move the $X, Y, Z$ reference system to the $x, y, z$, we need one rotation of $\sigma$ around the third axis and one rotation of $\rho$ around the second axis.

The rotation coefficients are given by the following formulas.

From the inertial orbital system to the angular momentum system:

$$
\begin{aligned}
& a_{x X}=\cos \sigma \cos \rho, \quad a_{x Y}=\sin \sigma \cos \rho, \quad a_{x Z}=-\sin \rho, \\
& a_{y X}=-\sin \sigma, \quad a_{y}=\cos \sigma, \quad a_{y}=0, \\
& a_{z X}=\cos \sigma \sin \rho, \quad a_{z Y}=\sin \sigma \sin \rho, \quad a_{z Z}=\cos \rho .
\end{aligned}
$$

From the body fixed system to the angular momentum system (type II Euler angles):

$$
\begin{gathered}
a_{x \xi}=\cos \phi \cos \psi-\sin \phi \sin \psi \cos \theta, \\
a_{x \eta}=-\cos \phi \sin \psi-\sin \phi \cos \psi \cos \theta, \quad a_{x \zeta}=\sin \theta \sin \phi, \\
a_{y \xi}=\sin \phi \cos \psi+\cos \phi \sin \psi \cos \theta, \\
a_{y \eta}=-\sin \phi \sin \psi+\cos \phi \cos \psi \cos \theta, \quad a_{y \zeta}=-\sin \theta \cos \phi, \\
a_{z \xi}=\sin \theta \sin \psi, \quad a_{z \eta}=\sin \theta \cos \psi \quad a_{z \zeta}=\cos \theta .
\end{gathered}
$$

Notice that the $a_{z *}$ terms are the only ones that do not depend on the angle $\phi$. 


\subsection{The rotation dynamical system}

We can describe the rotational dynamics of the rigid body using the angles $\theta$, $\phi, \psi$, which describe the rigid motion with respect to the angular momentum system, the angles $\rho, \sigma$ and the angular momentum magnitude $L$, which describes the inertial motion of the angular momentum vector $\mathbf{L}$.

In the unperturbed problem, i.e., with zero torque, we have an analytical, periodic, solution for the angles $\theta, \phi, \psi$ (Euler-Poinsot solution) in terms of elliptic functions, while $\rho, \sigma, L$ are constants, see [MacMillan 1936].

According to [Neishtadt et al. 2003], the rotation dynamical system is given by

$$
\begin{gathered}
\frac{d \theta}{d t}=L \sin \theta \sin \psi \cos \psi\left(\frac{1}{A}-\frac{1}{B}\right)+\frac{1}{L}\left[\left(M_{\xi} \sin \psi+M_{\eta} \cos \psi\right) \cos \theta-M_{\zeta} \sin \theta\right], \\
\frac{d \phi}{d t}=L\left(\frac{\sin ^{2} \psi}{A}+\frac{\cos ^{2} \psi}{B}\right)-\frac{M_{x}}{L} \cos \phi \cot \theta-\frac{M_{y}}{L}(\cot \rho+\sin \phi \cot \theta), \\
\frac{d \psi}{d t}=L \cos \theta\left(\frac{1}{C}-\frac{\sin ^{2} \psi}{A}-\frac{\cos ^{2} \psi}{B}\right)+\frac{M_{\xi} \cos \psi-M_{\eta} \sin \psi}{L \sin \theta}, \\
\frac{d \rho}{d t}=\frac{M_{x}}{L}, \quad \frac{d \sigma}{d t}=\frac{M_{y}}{L \sin \rho}, \quad \frac{d L}{d t}=M_{z},
\end{gathered}
$$

where $\left(M_{x}, M_{y}, M_{z}\right)$ and $\left(M_{\xi}, M_{\eta}, M_{\zeta}\right)$ are the components of the torque with respect to the angular momentum and the body-fixed systems. Note that these equations are singular for $\theta=0, \pi$ and $\rho=0, \pi$, and thus we cannot use them to integrate for principal axis rotation around the maximum momentum of inertia. However we can change coordinate system between the angular momentum system and the body fixed one, using Euler I angles instead of Euler II. With these coordinates the equations are singular for $\theta^{\prime}= \pm \pi / 2$, and the following discussion about the averaged dynamics follows in exactly the same way.

Another important equation is the one for the so called dynamic inertia

$$
\begin{gathered}
I_{D}=\frac{L^{2}}{2 T}=\left(\frac{\sin ^{2} \theta \sin ^{2} \psi}{A}+\frac{\sin ^{2} \theta \cos ^{2} \psi}{B}+\frac{\cos ^{2} \theta}{C}\right)^{-1} \\
\frac{d I_{D}}{d t}=-\frac{2 I_{D}}{L}\left[\frac{\left(I_{D}-A\right)}{A} a_{z \xi} M_{\xi}+\frac{\left(I_{D}-B\right)}{B} a_{z \eta} M_{\eta}+\frac{\left(I_{D}-C\right)}{C} a_{z \zeta} M_{\zeta}\right]
\end{gathered}
$$

where $T$ is the rotational kinetic energy. Note that $I_{D}$ is constant in the unperturbed motion. If $A<I_{D}<B$ we say that the body is in Long Axis Mode $(L A M)$ rotation as the angular velocity revolves about the axis of minimum inertia, if $B<I_{D}<C$ we say that it is in Short Axis Model $(S A M)$ rotation, as the angular velocity revolves about the axis of maximum 
moment of inertia. When $I_{D}=A, C$ the body is in principal axis rotation around the axes $\xi$ and $\zeta$. In general, we say that the body is in $L A M_{+}$or $L A M_{-}$if the rotation axis is closer to the axis $\xi$ in the positive or negative direction. In an analogous way we say that it is in a $S A M_{+}$or $S A M_{-}$if the rotation axis is closer to the axis $\zeta$ in the positive or negative direction.

When $I_{D}=B$ the body is in principal axis rotation around the $\eta$-axis or its angular velocity moves along a heteroclinic connection between rotations about the $\eta$-axis in opposite directions. $I_{D}$ is an important parameter because it tells us how the body is in intermediate principal axis rotation.

\subsection{The YORP torque}

We first consider a continous model for the rigid body. Let $d \mathbf{S}=d S \mathbf{n}$ be the normal to a surface element of the body, and let $\mathbf{r}$ be its position with respect to the center of mass.

We assume in this first discussion that the body has zero thermal conductivity, which means that the infrared thermal emission takes place immediately after the solar photons have hit the body's surface. We also assume that the body is moving on a Keplerian orbit around the Sun, with semimajor axis $a$, eccentricity $e$ and orbital period $T_{\text {orb }}$. Thus we can model the thermal YORP torque, due to the thermal radiation force normal to the body's surface, by the formula ([Rubincam 2000]):

$$
\mathbf{M}=P(R) a_{2} \oint f d S \mathbf{d}
$$

where $a_{2}=2 / 3\left(1-p_{v}\right)$ and $p_{v}$ is the albedo of the body, $f$ is the illumination function that is equal to 0 when the surface element is not illuminated by the Sun, and it is equal to $\mathbf{n} \cdot \mathbf{u}$ when it is, $\mathbf{u}$ is the position of the Sun in the body fixed system,

$$
\mathbf{d}=\mathbf{n} \times \mathbf{r}
$$

and

$$
P(R)=\frac{G_{1}}{a^{2}\left(1-e^{2}\right)^{2}}(1+e \cos \nu)^{2}
$$

where $G_{1} \cong 7.5 \times 10^{23} \mathrm{~kg} \mathrm{~km} / \mathrm{d}^{2}$ (see [Scheeres 2007]) is such that $P(R)=$ $G_{1} / R^{2}$ is the solar radiation pressure at $R \mathrm{~km}$ from the Sun. Finally $\nu$ is the true anomaly.

The strongest limitation of this analytical approach is modelling the illumination function $f$. If we do not take into account possible "selfshadowing" of the body, which means that the Sun is not illuminating a portion of the surface even if it is above its horizon (e.g. for a "bananashape" asteroid), we can express the illumination function $f$ analytically by:

$$
f=\frac{1}{2}(|\mathbf{n} \cdot \mathbf{u}|+\mathbf{n} \cdot \mathbf{u}) .
$$


So a first approximation of this model is to not consider "self-shadowing" bodies. Putting $\mathbf{n} \cdot \mathbf{u}=\cos z$, we can express $f$ by its Fourier series expansion:

$$
\begin{gathered}
f(z)=\frac{1}{2}(|\cos z|+\cos z)=\frac{a_{0}}{2}+\sum_{n=1}^{\infty} a_{n} \cos n z \\
a_{n}=\frac{1}{\pi} \int_{-\pi}^{\pi} f(z) \cos n z d z .
\end{gathered}
$$

Truncating the series to its fourth order term we have:

$$
\begin{aligned}
f & =\frac{1}{\pi}+\frac{1}{2} \cos z+\frac{2}{3 \pi} \cos 2 z-\frac{2}{15 \pi} \cos 4 z+\ldots \\
& =\frac{1}{5 \pi}+\frac{1}{2}(\mathbf{n} \cdot \mathbf{u})+\frac{12}{5 \pi}(\mathbf{n} \cdot \mathbf{u})^{2}-\frac{16}{15 \pi}(\mathbf{n} \cdot \mathbf{u})^{4}+\ldots
\end{aligned}
$$

To simplify the analytical results we approximate $f$ by using its second order expansion (following [Mysen 2008]):

$$
\begin{aligned}
f & =\frac{1}{\pi}+\frac{1}{2} \cos z+\frac{2}{3 \pi} \cos 2 z+\ldots \\
& =\frac{1}{3 \pi}+\frac{1}{2}(\mathbf{n} \cdot \mathbf{u})+\frac{4}{3 \pi}(\mathbf{n} \cdot \mathbf{u})^{2}+\ldots
\end{aligned}
$$

However, it is not obvious how much the higher order terms or the effect of self-shadowing will modify the dynamics, and how strong this effect could be for arbitrary shapes. It is then possible that this approximation is not accurate enough, and that we will need to consider additional terms of the Fourier series in the future. We will come back to this problem later, when we discuss some generalizations. We also notice that, from elementary calculus theory, for a generic regular shape we have the following result (see for example [Mysen 2007]):

$$
\oint d S \mathbf{d}=0
$$

then the constant term in the Fourier series approximation of $f$ does not contribute to the thermal YORP torque $\mathbf{M}$ given by formula (3).

Since we also would like to perform some numerical experiments, it is more convenient to model the shape of the rigid body in a discrete way, considering that its surface is formed by $N$ triangular facets. Let $A_{i}$ be the area of the $i$-th facet, $\mathbf{r}_{i}$ the position of the facet's center with respect to the center of mass of the body, and $\mathbf{n}_{i}$ the unit vector normal to the facet.

Then we have the discrete formula for the thermal YORP, without thermal conductivity:

$$
\mathbf{M}=P(R) a_{2} \sum_{i}^{N} f_{i} A_{i} \mathbf{d}_{i}
$$


where in this case

$$
f_{i}=\frac{1}{3 \pi}+\frac{1}{2}\left(\mathbf{n}_{i} \cdot \mathbf{u}\right)+\frac{4}{3 \pi}\left(\mathbf{n}_{i} \cdot \mathbf{u}\right)^{2}
$$

and

$$
\mathbf{d}_{i}=\mathbf{n}_{i} \times \mathbf{r}_{i}
$$
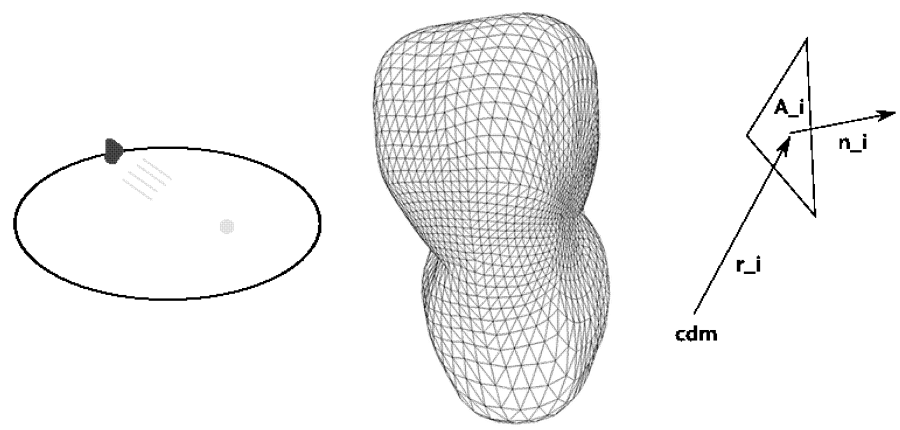

Figure 2: A simple cartoon showing an asteroid orbiting around the Sun and hit by the solar photons, and a discrete shape model obtained by a triangulation of the body's surface.

Notice that while the torque can be directly integrated over a single facet for a given obliquity (see [Scheeres 2007]) the resulting formula cannot be subsequently averaged, or at least not easily. The Mysen approximation in (7) makes the resultant torque amenable to further averaging and enables our current result.

\subsection{Dissipation of Energy}

For completeness, in this subsection we set up the equations of the rotational dynamics with a small dissipation of energy. If we do not assume a perfect rigid body model, we have to consider energy dissipation. Frictional internal processes tend to dissipate energy of the fluctuating strain field in the body. The fluctuating strain energy is nonzero when the body is in non-principal axis rotation as the rotational acceleration is time varying in the body-fixed frame.

We can model this effect by adding the following torque to the equations of motion, where we discriminate between SAM and LAM rotation states (see [Vokrouhlicky et al. 2007]): 
$S A M$ case

$$
\mathbf{M}_{d}=\mp \frac{\varrho a_{e q}^{2}}{\mu Q C^{3}} L^{3} \sin \theta\left(b_{0}+b_{2} \cos ^{2} \theta\right) \mathbf{L} \times\left(\frac{\mathbf{L} \times \hat{\zeta}}{L \sin \theta}\right)
$$

where $\varrho$ is the bulk density, $\mu$ is the rigidity, $Q$ the quality factor, $a_{e q}$ the (averaged) equatorial radius, and $b_{0}, b_{2}$ are the same known constants given in [Vokrouhlicky et al. 2007]. The sign - is in the $S A M_{+}$case, the sign + is in the $S A M_{-}$case.

$L A M$ case

$$
\mathbf{M}_{d}= \pm \frac{\varrho a_{e q}^{2}}{\mu Q A^{3}} L^{3} \sin \theta^{\prime}\left(b_{0}+b_{2} \cos ^{2} \theta^{\prime}\right) \mathbf{L} \times\left(\frac{\mathbf{L} \times \hat{\xi}}{L \sin \theta^{\prime}}\right)
$$

where $\theta^{\prime}$ is the angle between $\mathbf{L}$ and $\hat{\xi}$. The sign + in in the $L A M_{+}$case, the sign - is in the $L A M_{-}$case.

Values for the physical parameters can be (e.g. in [Sharma et al. 2005]):

$$
\varrho=2.5 \mathrm{~g} / \mathrm{cm}^{3} \quad \mu Q=5 \times 10^{12} \mathrm{~g} / \mathrm{cm} \mathrm{s}^{2} .
$$

\section{Analytical formulas for the secular solution}

Since the torque $\mathbf{M}$ can be considered a "small torque" with respect to the angular momentum, we can apply a first order perturbation, averaged theory as described in [Arnold et al. 1998], where a tool is introduced for the long term averaged analysis of a certain kind of dynamical system. Following closely the notation of [Arnold et al. 1998], we indicate with $I$ the quantities $\rho, \sigma, L$ and $I_{D}$, which are constants in the unperturbed rotational motion (slow variables in the perturbed motion), and with $\varphi$ the coordinates on the 4-dimensional torus $\mathbf{T}^{4}$ parametrizing the unperturbed periodic EulerPoinsot rotational motion and the Keplerian orbital motion (fast variables).

The general formulas for this problem are then of the form

$$
\dot{I}=0 \quad \dot{\varphi}=\omega(I)
$$

for the unperturbed Euler-Poinsot rotational motion and the Keplerian orbital motion, while they are

$$
\dot{I}=\epsilon f(I, \varphi, \epsilon) \quad \dot{\varphi}=\omega(I)+\epsilon g(I, \varphi, \epsilon)
$$

for the perturbed rotational motion in the small parameter $\epsilon$, ratio of the YORP torque and the angular momentum. Then, we can average over the 
unperturbed Euler-Poinsot motion and over the Keplerian orbital motion through the angles $\varphi$ by using Arnold's formula:

$$
\dot{\bar{I}}=\epsilon F(\bar{I}) \quad F(\bar{I})=(2 \pi)^{-4} \oint_{\mathbf{T}^{4}} f(\bar{I}, \varphi, 0) d \varphi
$$

where $\bar{I}$ indicates the first order averaged quantities $\bar{\rho}, \bar{\sigma}, \bar{L}$ and $\bar{I}_{D}$.

The difference between the true solution $I$ and the secular solution $\bar{I}$ consists in short periodic oscillations $I_{p}=I-\bar{I}$. Exploiting the fact that the oscillations of the function $I_{p}$ have finite non zero amplitude, we will able to reach some interesting conclusion on the behaviour of the true solution (section 5).

In the following, in order to obtain the secular evolutionary equations, we compute the first order averaged equations for the slow variables $I$ of the system, constants in the Euler-Poinsot motion. Then we verify the agreement with the full solution obtained from system (1) over short time spans. In the next sections we study the averaged solution $\bar{I}$ over long time spans, with the intent to capture the generic evolution of the dynamical system.

According to the previous section 2, not considering the dissipation of energy, in order to compute the averaged equations for the quantities $\rho, \sigma$, $L$ and $I_{D}$ we can write the YORP torque in the angular momentum frame as:

$$
\begin{aligned}
& M_{x}=P(R) \frac{a_{2}}{2} \oint d S d_{x}(\mathbf{n} \cdot \mathbf{u})+P(R) \frac{4 a_{2}}{3 \pi} \oint d S d_{x}(\mathbf{n} \cdot \mathbf{u})^{2}, \\
& M_{y}=P(R) \frac{a_{2}}{2} \oint d S d_{y}(\mathbf{n} \cdot \mathbf{u})+P(R) \frac{4 a_{2}}{3 \pi} \oint d S d_{y}(\mathbf{n} \cdot \mathbf{u})^{2}, \\
& M_{z}=P(R) \frac{a_{2}}{2} \oint d S d_{z}(\mathbf{n} \cdot \mathbf{u})+P(R) \frac{4 a_{2}}{3 \pi} \oint d S d_{z}(\mathbf{n} \cdot \mathbf{u})^{2},
\end{aligned}
$$

where $(\mathbf{n} \cdot \mathbf{u})=u_{x} n_{x}+u_{y} n_{y}+u_{z} n_{z}$,

$$
\begin{array}{cc}
(\mathbf{n} \cdot \mathbf{u})^{2}=u_{x}^{2} n_{x}^{2}+u_{y}^{2} n_{y}^{2}+u_{z}^{2} n_{z}^{2}+2 u_{x} u_{y} n_{x} n_{y}+2 u_{x} u_{z} n_{x} n_{z}+2 u_{y} u_{z} n_{y} n_{z}, & \\
d_{x}=a_{x \xi} d_{\xi}+a_{x \eta} d_{\eta}+a_{x \zeta} d_{\zeta}, & n_{x}=a_{x \xi} n_{\xi}+a_{x \eta} n_{\eta}+a_{x \zeta} n_{\zeta}, \\
d_{y}=a_{y \xi} d_{\xi}+a_{y \eta} d_{\eta}+a_{y} d_{\zeta}, & n_{y}=a_{y \xi} n_{\xi}+a_{y \eta} n_{\eta}+a_{y} n_{\zeta}, \\
d_{z}=a_{z \xi} d_{\xi}+a_{z \eta} d_{\eta}+a_{z \zeta} d_{\zeta}, & n_{z}=a_{z \xi} n_{\xi}+a_{z \eta} n_{\eta}+a_{z \zeta} n_{\zeta}, \\
u_{x}=-a_{x X} \sin \nu-a_{x Z} \cos \nu, u_{y}=-a_{y X} \sin \nu, & u_{z}=-a_{z X} \sin \nu-a_{z Z} \cos \nu .
\end{array}
$$

At this point we need to average $M_{x}, M_{y}, M_{z}$ over the unperturbed rotational motion and over the orbit. Assuming no resonance between the rotational and the orbital motion, we can average separately over them. This 
computation is done following the same procedure of [Neishtadt et al. 2003], where such resonances are considered (in their section 3.6). We are not considering these resonances in this paper, and reserve their consideration to future work.

Indicating with $<\cdot>_{o}$ and $<\cdot>_{e}$ the averaged quantities over the orbital motion and the unperturbed rotational motion, we have the following results:

$$
\begin{array}{rl}
<P(R)>_{o} & =\frac{1}{T} \int_{0}^{T} P(R) d t=\frac{\left(1-e^{2}\right)^{3 / 2}}{2 \pi} \int_{0}^{2 \pi} \frac{P(R(\nu)) d \nu}{(1+e \cos \nu)^{2}}=\frac{G_{1}}{a^{2} \sqrt{\left(1-e^{2}\right)}} \\
<P & P(R) \sin \nu>_{o}=<P(R) \cos \nu>_{o}=<P(R) \sin \nu \cos \nu>_{o}=0 \\
<P(R) \sin ^{2} \nu>_{o}=<P(R) \cos ^{2} \nu>_{o}=\frac{G_{1}}{2 a^{2} \sqrt{\left(1-e^{2}\right)}}
\end{array}
$$

and then

$$
<<P(R) \oint d S \mathbf{d}(\mathbf{n} \cdot \mathbf{u})>_{e}>_{o}=0 .
$$

We still have to compute:

$$
<a_{* *}>_{e} ; \quad<a_{* *} a_{* *}>_{e} ; \quad<a_{* *} a_{* *} a_{* *}>_{e} .
$$

To compute these we will assume that there is no resonance between the unperturbed periodic motion of the angles $\phi$ and $\psi, \theta$ (see again [Neishtadt et al. 2003] section 3.6). Noting that the period of the angle $\psi$ is twice the period of $\theta$, it is possible to average over the angle $\phi$ separately, dropping out the dependence on $\phi$ and expressing all the averaged quantities in terms of the $z$ - components $<a_{z *}>_{e},<a_{z *} a_{z *}>_{e}$ and $<a_{z *} a_{z *} a_{z *}>_{e}$ only.

However, the time dependance of the quantities $a_{* *}$ through the (unperturbed) angle $\phi$ is much more complicated than the one through the angles $\theta, \psi$. Let's indicate with $P_{\phi}$ the period of the unperturbed angle $\phi$, then we will use the following approximation to average over its motion:

$$
<a_{* *}>_{\phi}=\frac{1}{P_{\phi}} \int_{0}^{P_{\phi}} a_{* *}(\phi(t)) d t \cong \frac{1}{2 \pi} \int_{0}^{2 \pi} a_{* *}(\phi) d \phi=a_{* *}(\theta, \psi) .
$$

The time dependence through the angles $\theta, \psi$ instead is simpler and is based on the elliptic functions $s n, c n, d n$. Through them we can average over their motion using an integral over time:

$$
<a_{* *}>_{e}=\frac{1}{P_{\theta \psi}} \int_{0}^{P_{\theta \psi}} a_{* *}(\theta(t), \psi(t)) d t
$$

where $P_{\theta \psi}$ is the suitable period. 
Notice that because the functions $\sin \phi$ and $\cos \phi$ average to zero we can conclude that the quantities $\left.<a_{x *}\right\rangle_{e}$ and $\left.<a_{y *}\right\rangle_{e}$ average to zero.

We also have to compute the products $<a_{* *} a_{* *} a_{* *}>_{e}$ that appear in the term with $(\mathbf{n} \cdot \mathbf{u})^{2}$, where we have to compute the quantities $<d_{*} n_{*} n_{*}>$ with all the possible combinations. The functions $\sin ^{3} \phi, \cos ^{3} \phi, \sin ^{2} \phi \cos \phi$, $\sin \phi \cos ^{2} \phi$ all average to zero. Then, with the assumption of no resonance, the products $<a_{* *} a_{* *} a_{* *}>_{e}$ involving only the $x-$ and $y$-components and the products $<a_{x *} a_{z *} a_{z *}>_{e}$ and $<a_{y *} a_{z *} a_{z *}>_{e}$ are zero. The remaining terms that we have to compute are:

$<a_{x *} a_{x *} a_{z *}>_{e}, \quad<a_{x *} a_{y *} a_{z *}>_{e}, \quad<a_{y *} a_{y *} a_{z *}>_{e}, \quad<a_{z *} a_{z *} a_{z *}>_{e}$.

Using the notation $\bar{X}$ instead of $\langle X\rangle$ when the meaning is clear, we have:

$$
\bar{M}_{*}=<<P(R) \frac{4 a_{2}}{3 \pi} \oint d S d_{*}(\mathbf{n} \cdot \mathbf{u})^{2}>_{e}>_{o}
$$

for $*=x, y, z$. A list with the computed averaged factors $<a_{* *} a_{* *} a_{* *}>_{e}$ used in the following is given in the Appendix.

\subsection{Quantities averaged over the orbital motion}

According to the first part of this section, the terms containing the true anomaly $\nu$ are averaged over one orbital period to yield:

$$
\begin{aligned}
\overline{P(R)} & =\frac{G_{1}}{a^{2} \sqrt{\left(1-e^{2}\right)}}, \quad \overline{P(R) u_{x}^{2}}=\frac{\overline{P(R)}}{2}\left(\cos ^{2} \sigma \cos ^{2} \rho+\sin ^{2} \rho\right), \\
\overline{P(R) u_{y}^{2}} & =\frac{\overline{P(R)}}{2} \sin ^{2} \sigma, \quad \overline{P(R) u_{z}^{2}}=\frac{\overline{P(R)}}{2}\left(\cos ^{2} \sigma \sin ^{2} \rho+\cos ^{2} \rho\right), \quad(11) \\
\overline{P(R) u_{x} u_{z}} & =-\frac{\overline{P(R)}}{2} \sin ^{2} \sigma \sin \rho \cos \rho, \quad \overline{P(R) u_{y} u_{z}}=-\frac{\overline{P(R)}}{2} \sin \sigma \cos \sigma \sin \rho .
\end{aligned}
$$

\subsection{Factors averaged over the rotational motion}

Putting together all the previous results, we obtain the following averaged factors over the unperturbed rotational motion:

In the SAM case:

$$
\begin{gathered}
\overline{d_{z}}=d_{\zeta}<a_{z \zeta}>_{e} \\
\overline{d_{z} n_{z}^{2}}=<a_{z \zeta}^{3}>_{e} d_{\zeta} n_{\zeta}^{2}+<a_{z \xi}^{2} a_{z \zeta}>_{e}\left(2 d_{\xi} n_{\xi} n_{\zeta}+d_{\zeta} n_{\xi}^{2}\right)+ \\
+<a_{z \eta}^{2} a_{z \zeta}>_{e}\left(2 d_{\eta} n_{\eta} n_{\zeta}+d_{\zeta} n_{\eta}^{2}\right)
\end{gathered}
$$




$$
\begin{aligned}
\overline{d_{x} n_{y} n_{z}} & =\frac{1}{2}\left(<a_{z \zeta}^{2}>_{e}-<a_{z \eta}^{2}>_{e}\right) d_{\xi} n_{\eta} n_{\zeta}+ \\
& +\frac{1}{2}\left(<a_{z \xi}^{2}>_{e}-<a_{z \zeta}^{2}>_{e}\right) d_{\eta} n_{\zeta} n_{\xi}+ \\
& +\frac{1}{2}\left(<a_{z \eta}^{2}>_{e}-<a_{z \xi}^{2}>_{e}\right) d_{\zeta} n_{\xi} n_{\eta} .
\end{aligned}
$$

\section{In the LAM case:}

$$
\begin{gathered}
\overline{d_{z}}=d_{\xi}<a_{z \xi}>_{e} \\
\overline{d_{z} n_{z}^{2}}=<a_{z \xi}^{3}>_{e} d_{\xi} n_{\xi}^{2}+<a_{z \xi} a_{z \zeta}^{2}>_{e}\left(2 d_{\zeta} n_{\xi} n_{\zeta}+d_{\xi} n_{\zeta}^{2}\right)+ \\
+<a_{z \xi} a_{z \eta}^{2}>_{e}\left(2 d_{\eta} n_{\eta} n_{\xi}+d_{\xi} n_{\eta}^{2}\right) \\
\overline{d_{x} n_{y} n_{z}}=\frac{1}{2}\left(<a_{z \zeta}^{2}>_{e}-<a_{z \eta}^{2}>_{e}\right) d_{\xi} n_{\eta} n_{\zeta}+ \\
+\frac{1}{2}\left(<a_{z \xi}^{2}>_{e}-<a_{z \zeta}^{2}>_{e}\right) d_{\eta} n_{\zeta} n_{\xi}+ \\
+\frac{1}{2}\left(<a_{z \eta}^{2}>_{e}-<a_{z \xi}^{2}>_{e}\right) d_{\zeta} n_{\xi} n_{\eta}
\end{gathered}
$$

\section{In both cases:}

$$
\begin{array}{cl}
\overline{d_{x} n_{x} n_{z}}=-\frac{1}{2} \overline{d_{z} n_{z}^{2}}, \quad \overline{d_{z} n_{x}^{2}}=\frac{1}{2}\left(\overline{d_{z}}-\overline{d_{z} n_{z}^{2}}\right), \quad \overline{d_{z} n_{y}^{2}}=\overline{d_{z} n_{x}^{2}}, \\
\overline{d_{y} n_{y} n_{z}}=\overline{d_{x} n_{x} n_{z}}, \quad \overline{d_{y} n_{x} n_{z}}=-\overline{d_{x} n_{y} n_{z}}, \quad \overline{d_{z} n_{x} n_{y}}=0 .
\end{array}
$$

\subsection{Averaged equations}

Using the previous formulas and notation, we can write the averaged equations for the angular momentum vector and the dynamic inertia as:

$$
\begin{gathered}
\frac{d \bar{\rho}}{d t}=\frac{\bar{M}_{x}}{\bar{L}}, \quad \frac{d \bar{\sigma}}{d t}=\frac{\bar{M}_{y}}{\bar{L} \sin \bar{\rho}}, \quad \frac{d \bar{L}}{d t}=\bar{M}_{z} \\
\frac{d \bar{I}_{D}}{d t}=-\frac{2 \bar{I}_{D}}{\bar{L}}\left[\frac{\left(\bar{I}_{D}-A\right)}{A} \overline{a_{z \xi} M_{\xi}}+\frac{\left(\bar{I}_{D}-B\right)}{B} \overline{a_{z \eta} M_{\eta}}+\frac{\left(\bar{I}_{D}-C\right)}{C} \overline{a_{z \zeta} M_{\zeta}}\right],
\end{gathered}
$$

where

$$
\begin{gathered}
\bar{M}_{x}=\frac{8 a_{2}}{3 \pi}\left[\overline{P(R) u_{x} u_{z}} \oint d S \overline{d_{x} n_{x} n_{z}}+\overline{P(R) u_{y} u_{z}} \oint d S \overline{d_{x} n_{y} n_{z}}\right]= \\
=\frac{4 a_{2} \overline{P(R)}}{3 \pi}\left[\frac{1}{2} \sin ^{2} \bar{\sigma} \sin \bar{\rho} \cos \bar{\rho} \oint d S \overline{d_{z} n_{z}^{2}}-\sin \bar{\sigma} \cos \bar{\sigma} \sin \bar{\rho} \oint d S \overline{d_{x} n_{y} n_{z}}\right],
\end{gathered}
$$




$$
\begin{gathered}
\bar{M}_{y}=\frac{8 a_{2}}{3 \pi}\left[\overline{P(R) u_{x} u_{z}} \oint d S \overline{d_{y} n_{x} n_{z}}+\overline{P(R) u_{y} u_{z}} \oint d S \overline{d_{y} n_{y} n_{z}}\right]= \\
=\frac{4 a_{2} \overline{P(R)}}{3 \pi}\left[\frac{1}{2} \sin \bar{\sigma} \cos \bar{\sigma} \sin \bar{\rho} \oint d S \overline{d_{z} n_{z}^{2}}+\sin ^{2} \bar{\sigma} \sin \bar{\rho} \cos \bar{\rho} \oint d S \overline{d_{x} n_{y} n_{z}}\right] \\
\bar{M}_{z}=\frac{4 a_{2}}{3 \pi}\left[\overline{P(R) u_{x}^{2}} \oint d S \overline{d_{z} n_{x}^{2}}+\overline{P(R) u_{y}^{2}} \oint d S \overline{d_{z} n_{y}^{2}}+\overline{P(R) u_{z}^{2}} \oint d S \overline{d_{z} n_{z}^{2}}\right]= \\
=\frac{2 a_{2} \overline{P(R)}}{3 \pi}\left(\frac{1}{2}-\frac{3}{2} \sin ^{2} \bar{\rho} \sin ^{2} \bar{\sigma}\right) \oint d S \overline{d_{z} n_{z}^{2}}
\end{gathered}
$$

and

$$
\begin{gathered}
\overline{a_{z *} M_{*}}=\frac{4 a_{2}}{3 \pi}\left[\overline{P(R) u_{x}^{2}} \oint d S \overline{a_{z *} d_{*} n_{x}^{2}}+\overline{P(R) u_{y}^{2}} \oint d S \overline{a_{z *} d_{*} n_{y}^{2}}+\overline{P(R) u_{z}^{2}} \oint d S \overline{a_{z *} d_{*} n_{z}^{2}}\right]= \\
=\frac{2 a_{2} \overline{P(R)}}{3 \pi}\left(\frac{1}{2}-\frac{3}{2} \sin ^{2} \bar{\rho} \sin ^{2} \bar{\sigma}\right) \oint d S \overline{a_{z *} d_{*} n_{z}^{2}}
\end{gathered}
$$

where $*=\xi, \eta, \zeta$, and for example $\overline{a_{z \xi} d_{\xi} n_{x}^{2}}$ is obtained from $\overline{d_{z} n_{x}^{2}}$ keeping only the terms containing $a_{z \xi}$ and $d_{\xi}$.

\subsection{The obliquity}

In order to simplify the discussion we introduce the most important quantity that describes the direction of the angular momentum vector in the inertial space: the obliquity $\delta \in[0, \pi]$, which is the angle between the normal to the orbit $\mathbf{Y}$ and the angular momentum $\mathbf{L}$.

This angle is related to the angles $\rho$ and $\sigma$ by the equation:

$$
\cos \delta=\sin \rho \sin \sigma
$$

and its dynamics is given by the differential equation:

$$
\dot{\delta}=-\frac{1}{\sin \delta}\left(\frac{M_{x}}{L} \cos \rho \sin \sigma+\frac{M_{y}}{L} \cos \sigma\right) .
$$

In order to describe the secular dynamics of the obliquity, as we have done for the angles $\rho$ and $\sigma$, we derive the first order averaged equation for $\delta$ :

$$
\dot{\bar{\delta}}=-\frac{1}{\sin \bar{\delta}}\left(\frac{\bar{M}_{x}}{\bar{L}} \cos \bar{\rho} \sin \bar{\sigma}+\frac{\bar{M}_{y}}{\bar{L}} \cos \bar{\sigma}\right)
$$

and after some computation:

$$
\dot{\bar{\delta}}=-\frac{2 a_{2} \overline{P(R)}}{3 \pi \bar{L}} \sin \bar{\delta} \cos \bar{\delta} \oint d S \overline{d_{z} n_{z}^{2}} .
$$


We have reduced the dynamical system to the three fundamental quantities $\bar{\delta}, \bar{L}$ and $\bar{I}_{D}$. It is easy to see that, if we define the following quantities

$$
\begin{gathered}
\mathcal{D}_{1}=\mathcal{D}_{1}^{ \pm}\left(\bar{I}_{D}\right)=\oint d S \overline{d_{z} n_{z}^{2}} \\
\mathcal{D}_{3}=\mathcal{D}_{3}^{ \pm}\left(\bar{I}_{D}\right)= \\
=\frac{\bar{I}_{D}-A}{A} \oint d S \overline{a_{z \xi} d_{\xi} n_{z}^{2}}+\frac{\bar{I}_{D}-B}{B} \oint d S \overline{a_{z \eta} d_{\eta} n_{z}^{2}}+\frac{\bar{I}_{D}-C}{C} \oint d S \overline{a_{z \zeta} d_{\zeta} n_{z}^{2}},
\end{gathered}
$$

where the sign \pm depends on the mode $S A M / L A M_{ \pm}$, we can write the dynamical system in the simple form:

$$
\left\{\begin{aligned}
\dot{\bar{I}}_{D} & =-\frac{a_{2} \overline{P(R)}}{3 \pi} \frac{2 \bar{I}_{D}}{\bar{L}}\left(1-3 \cos ^{2} \bar{\delta}\right) \mathcal{D}_{3} \\
\dot{\bar{\delta}} & =-\frac{2 a_{2}}{P(R)} \sin \bar{\delta} \cos \bar{\delta} \mathcal{D}_{1} \\
\dot{\bar{L}} & =\frac{a_{2} \frac{2}{P(R)}}{3 \pi}\left(1-3 \cos ^{2} \bar{\delta}\right) \mathcal{D}_{1} .
\end{aligned}\right.
$$

The secular evolution of these quantities is governed by the qualitative behaviour of the functions $\mathcal{D}_{1}$ and $\mathcal{D}_{3}$. These functions depend on the parameter $\bar{I}_{D}$ through formulas (12)-(13) and the averaged factors in Appendix, and are regular functions in the open intervals $(A, B)$ and $(B, C)$. They have the dimension of a volume. We note that it can be shown that $\mathcal{D}_{1}=0$ for a triaxial ellipsoid shape (see for example [Mysen 2007]). More details about the zeros of these functions will be given in the following.

\subsection{Separation of variables and limitations}

Both the differential equations for $\bar{I}_{D}$ and $\bar{\delta}$ in the system (20) have the same dependance on $\bar{L}$ as a factor $1 / \bar{L}$. In particular the bigger $\bar{L}$ is the slower the variation of $\bar{I}_{D}$ and $\bar{\delta}$ is. Moreover, in all three equations we can separate the variables, yielding an integrable system for the averaged equations. Specifically, if we consider motion in the plane $\left(\bar{I}_{D}, \bar{\delta}\right)$, we can always find a (local) first integral.

More precisely, if $\bar{I}_{D}=x$ and $\bar{\delta}=y$, the first integral is:

$$
F(x, y)=e^{\frac{1}{6} \int_{x_{0}}^{x} \frac{\mathcal{D}_{1}\left(x^{\prime}\right)}{x^{\prime} \mathcal{D}_{3}\left(x^{\prime}\right)} d x^{\prime}-\int_{y_{0}}^{y} \frac{1-3 \cos ^{2} y^{\prime}}{3 \sin 2 y^{\prime}} d y^{\prime}}
$$

such that

$$
\frac{d}{d t} F(x, y)=F_{x}(x, y) \dot{x}+F_{y}(x, y) \dot{y}=0 .
$$

Using this result it is easy to describe the solution of the averaged system (see section 4). 
It also follows easily from equations (20) that there is a direct relation between $\bar{L}$ and $\bar{\delta}$ :

$$
\frac{\dot{\bar{\delta}}}{\dot{\bar{L}}}=-\frac{2}{\bar{L}} \frac{\sin \bar{\delta} \cos \bar{\delta}}{1-3 \cos ^{2} \bar{\delta}} \quad \Rightarrow \quad \bar{L}^{2}=K \sin ^{2} \bar{\delta} \cos \bar{\delta}
$$

where $K$ is a constant which depends on the initial conditions ${ }^{1}$.

However, we must notice here that the fact that this system has a first integral is a direct consequence of the separation of variables, which would not be true if we considered a better approximation for the illumination function $f$ (formula (4)), such as the approximation in formula (5), or incorporated self-shadowing of the body. This is clearly a limitation of the current analytical approach. However, modelling the self-shadowing of the body analytically is difficult, and considering a better approximation of $f$ involves the computation of many terms, perhaps too many for a compact description of the averaged equations. We are not considering these improvements to the model here, leaving this problem to be discussed in future works.

The possible qualitative evolution of this system is described in the next section, in which we only briefly discuss the generalizations mentioned above.

\subsection{Averaged equations with dissipation of energy}

For completeness, assuming that the dissipation torque is a small perturbation of the free rotational motion, we also obtain the first order averaged equations with dissipation of energy for the quantities $\bar{\rho}, \bar{\sigma}, \bar{L}, \bar{I}_{D}$ as in the case of the YORP torque.

It turns out that $\bar{\rho}, \bar{\sigma}$ and $\bar{L}$ are not affected by the dissipation, instead for $\bar{I}_{D}$ we have:

$$
\dot{\bar{I}}_{D}=-\frac{a_{2} \overline{P(R)}}{3 \pi} \frac{2 \bar{I}_{D}}{\bar{L}}\left(1-3 \cos ^{2} \bar{\delta}\right) \mathcal{D}_{3}+h_{d}\left(\bar{I}_{D}, \bar{L}\right)
$$

where, putting

$$
\mathcal{D}_{5}=\frac{\bar{I}_{D}-A}{A} c_{1}+\frac{\bar{I}_{D}-B}{B} c_{2}+\frac{\bar{I}_{D}-C}{C} c_{3},
$$

in the $S A M_{ \pm}$case we have:

$$
\begin{gathered}
h_{d}\left(\bar{I}_{D}, \bar{L}\right)= \pm 2 \frac{\varrho a_{e q}^{2}}{\mu Q C^{3}} \bar{L}^{3} \bar{I}_{D} \mathcal{D}_{5}\left(\bar{I}_{D}\right), \\
c_{1}=b_{0}<a_{z \xi}^{2} a_{z \zeta}>+b_{2}<a_{z \xi}^{2} a_{z \zeta}^{3}>, \quad c_{2}=b_{0}<a_{z \eta}^{2} a_{z \zeta}>+b_{2}<a_{z \eta}^{2} a_{z \zeta}^{3}>,
\end{gathered}
$$

\footnotetext{
${ }^{1}$ This formula has been derived by D. Vokrouhlicky (private discussion).
} 


$$
c_{3}=b_{0}\left(<a_{z \zeta}^{3}>-<a_{z \zeta}>\right)+b_{2}\left(<a_{z \zeta}^{5}>-<a_{z \zeta}^{3}>\right),
$$

and in the $L A M_{ \pm}$case:

$$
\begin{gathered}
h_{d}\left(\bar{I}_{D}, \bar{L}\right)=\mp 2 \frac{\varrho a_{e q}^{2}}{\mu Q A^{3}} \bar{L}^{3} \bar{I}_{D} \mathcal{D}_{5}\left(\bar{I}_{D}\right), \\
c_{1}=b_{0}\left(<a_{z \xi}^{3}>-<a_{z \xi}>\right)+b_{2}\left(<a_{z \xi}^{5}>-<a_{z \xi}^{3}>\right), \\
c_{2}=b_{0}<a_{z \xi} a_{z \eta}^{2}>+b_{2}<a_{z \xi}^{3} a_{z \eta}^{2}>, \quad c_{3}=b_{0}<a_{z \xi} a_{z \zeta}^{2}>+b_{2}<a_{z \xi}^{3} a_{z \zeta}^{2}>.
\end{gathered}
$$

It is straightforward to find that the sign of the quantity $h_{d}$ does not depend on the sign of the mode. Indeed, looking at the formulas for the averaged factors in Appendix, we see that the sign of the quantities $c_{i}$ changes if the sign of the mode changes, and so does the sign in front of the formula for $h_{d}$.

Intuitively, the averaged effect of the energy dissipation on the rotation would be to drive the body to principal axis $S A M$, so to drive $\bar{I}_{D}$ to the value $C$. Thus we expect $h_{d}$ to be always positive, which means $\mathcal{D}_{5}>0$ in the $S A M_{+}$mode and $\mathcal{D}_{5}<0$ in the $L A M_{+}$mode. Proving that analytically is not straightforward, but this is the case for all the shape models that we have tested (see section 6).

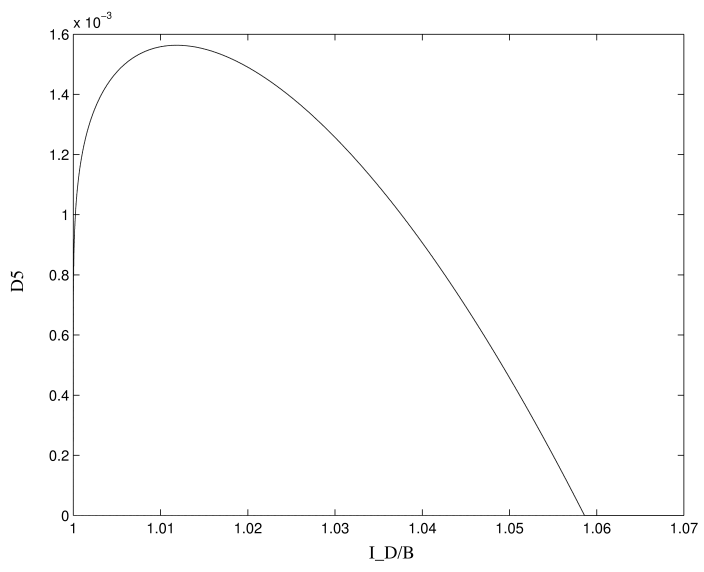

Figure 3: The $\mathcal{D}_{5}$ function in the $S A M_{+}$mode. We used the asteroid Toutatis shape model (see section 6.2 for more details about the model). 


\subsection{Comparison between full and averaged solution}

For example purposes we present some direct comparisons between numerical integrations and secular integration of the averaged equations. The full numerical integration consists of the implementation of the full dynamical system (1), while the secular integration consists of the separate implementation of the dynamical system (20) obtained by analytical averaging of (1).

To test the agreement between the full solution and the averaged solution we integrated both the equations using the asteroid Toutatis shape model, see section 6.2 for more details about the model, on a time span of $\Delta t \cong$ $20 T_{\text {orb }}$ with the following initial conditions (randomly chosen):

$$
\begin{aligned}
& \theta_{0}=30^{\circ}, \quad \phi_{0}=1^{\circ}, \quad \psi_{0}=50^{\circ}, \quad\left(\Rightarrow I_{D 0} \cong 1.36 \times 10^{13} \mathrm{~kg} \mathrm{~km}^{2}\right), \\
& \rho_{0}=40^{\circ}, \quad \sigma_{0}=90^{\circ} \quad\left(\Rightarrow \delta_{0} \cong 101.6^{\circ}\right), \quad L_{0}=1.08 \times 10^{13} \mathrm{~kg} \mathrm{~km}^{2} / \mathrm{d} .
\end{aligned}
$$

We recall that the obliquity is related to $\rho$ and $\sigma$ by $\cos \delta=\sin \rho \sin \sigma$.

The numerical integrator we use is the Matlab Ode45. The error control used is a RelTol of $10^{-12}$ and an AbsTol of $10^{-12}$.

Note the good agreement between the two over short time spans, which already are very computationally challenging for the full simulations. Based on a number of such comparisons we conclude that the secular equations properly represent the averaged motion.

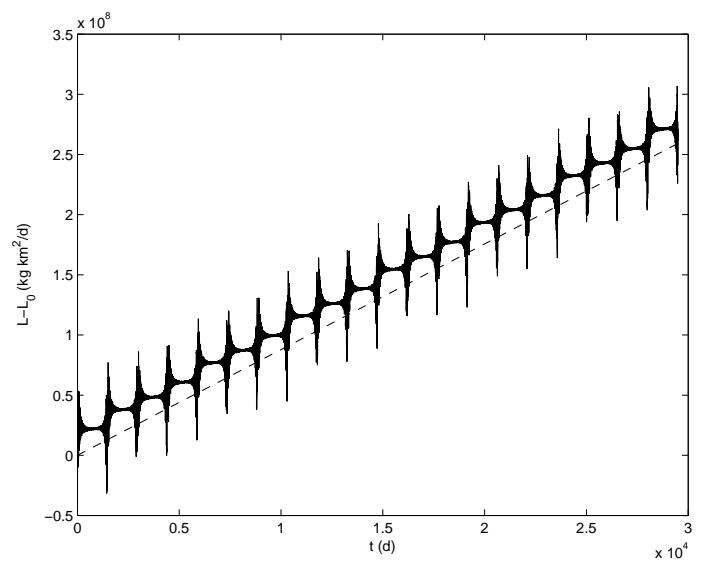

Figure 4: Comparison between the full solution (solid curve) and the averaged solution (dashed line) for the angular momentum magnitude $L-L_{0}$ (initial conditions (25)), plotted over a $\Delta t \cong 20 T_{\text {orb }}$ time span. 

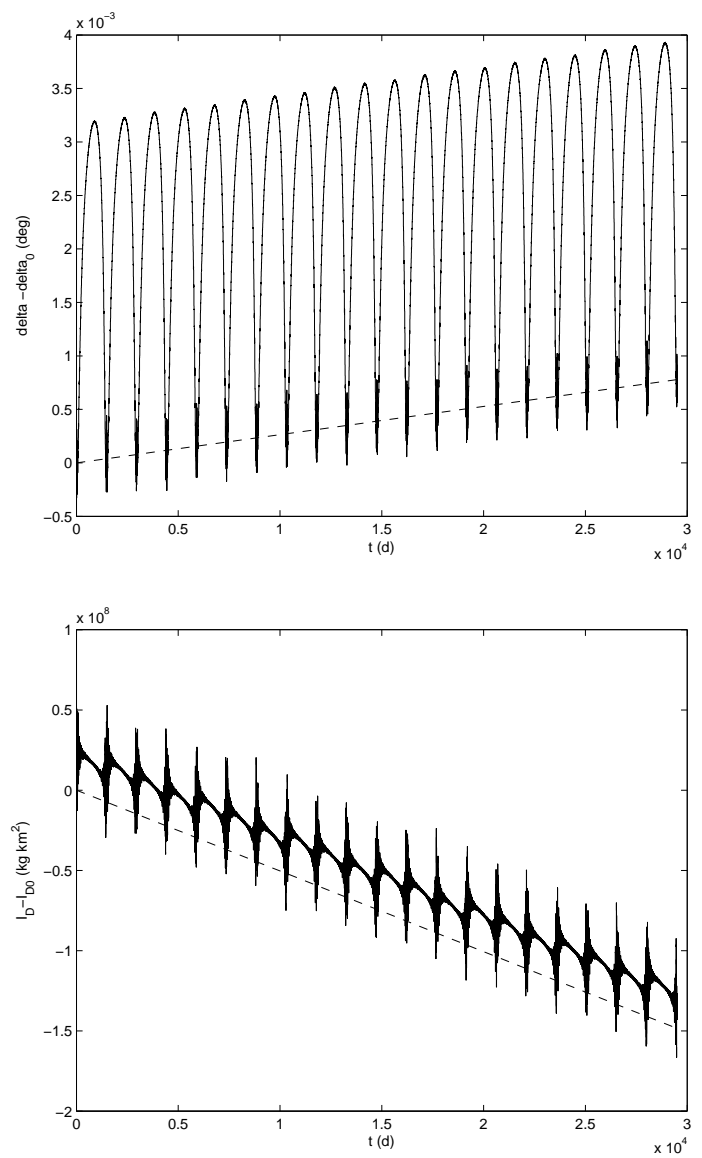

Figure 5: Comparison between the full solution (solid curve) and the averaged solution (dashed line), on the top the obliquity $\delta-\delta_{0}$, on the bottom the dynamic inertia $I_{D}-I_{D 0}$ (initial conditions (25)), both plotted over a $\Delta t \cong 20 T_{\text {orb }}$ time span. 


\section{Dynamics without dissipation}

We have seen that the principal quantities that describe the averaged behaviour of the rotational dynamics are the obliquity of the angular momentum, its magnitude, and the dynamic inertia parameter $\bar{I}_{D}$.

In the previous section we found that

$$
\dot{\bar{I}}_{D}=h\left(\bar{I}_{D}, \bar{\delta}, \bar{L}\right), \quad \dot{\bar{\delta}}=f\left(\bar{I}_{D}, \bar{\delta}, \bar{L}\right), \quad \dot{\bar{L}}=g\left(\bar{I}_{D}, \bar{\delta}\right),
$$

and that we can easily separate the variables. Thus it is possible to draw, at least qualitatively, the level curves $\dot{\bar{I}}_{D}=0, \dot{\bar{\delta}}=0$ and $\dot{\bar{L}}=0$ in the plane $\left(\bar{I}_{D}, \bar{\delta}\right)$. These curves, because of the separation of the variables, are straight lines, independent of the sign of the mode $S A M / L A M \pm$. Instead the parts of the plane in which $\dot{\bar{\delta}}, \dot{\bar{I}}_{D}$ and $\dot{\bar{L}}$ are positive or negative depend on the sign \pm of the mode $S A M / L A M \pm$, through the functions $\mathcal{D}_{k}^{ \pm}\left(\bar{I}_{D}\right)$.

If we know the rotation mode of the body, we can choose the sign \pm of the functions $\mathcal{D}_{k}=\mathcal{D}_{k}^{ \pm}\left(\bar{I}_{D}\right)$ (note that $\mathcal{D}_{k}^{-}=-\mathcal{D}_{k}^{+}$). Once the sign and the mode are set we can study the properties of these functions. In particular in the $S A M$ case we have to study $\mathcal{D}_{k}$ for $\bar{I}_{D} \in(B, C)$, in the $L A M$ case we have to study $\mathcal{D}_{k}$ for $\bar{I}_{D} \in(A, B)$.

This information, together with the level curves of the first integral of the system, gives us the Phase diagram structure, i.e. the parts of the plane $\left(\bar{I}_{D}, \bar{\delta}\right)$ in which the quantities $\dot{\bar{\delta}}, \dot{\bar{I}}_{D}, \dot{\bar{L}}$ are positive or negative and the qualitative behaviour of the solutions.

In order to qualitatively describe the dynamics, we will look for the stationary points of $\bar{\delta}, \bar{L}$ and $\bar{I}_{D}$.

Def. Let $\bar{x}$ be one of the quantities $\bar{I}_{D}, \bar{\delta}, \bar{L}$.

We define an equilibrium point for $\bar{x}$ a configuration of the body at a time $t_{0}$ such that

- $\dot{\bar{x}}(t)=0 \quad \forall t \geq t_{0}$.

We define a stationary point for $\bar{x}$ a configuration of the body at a time $t_{s}$ such that

- $\dot{\bar{x}}\left(t_{s}\right)=0$.

\subsection{Equilibrium points for the obliquity}

The behaviour of the obliquity $\bar{\delta}$ is described by the differential equation:

$$
\dot{\bar{\delta}}=-\alpha \sin \bar{\delta} \cos \bar{\delta} \mathcal{D}_{1},
$$

where $\alpha=2 a_{2} \overline{P(R)} / 3 \pi \bar{L}>0$. Then we deduce that $\dot{\bar{\delta}}=0$ if and only if: 
- $\bar{\delta}=\pi / 2$, i.e. $\mathbf{L}$ lies in the orbital plane

- $\bar{\delta}=0, \bar{\delta}=\pi$, i.e. $\mathbf{L}$ is normal to the orbital plane

- there is an $\bar{I}_{D}^{e}$ such that $\mathcal{D}_{1}\left(\bar{I}_{D}^{e}\right)=0$

In the first two cases $\dot{\bar{\delta}}$ is 0 whatever the value of $\bar{I}_{D}$, so the direction of the angular momentum remains fixed over time and we have three equilibrium points for $\bar{\delta}$.

In the third case note that $\lim _{I_{D} \rightarrow B} \mathcal{D}_{1}=0$ whatever the shape, but we will see that the case $\bar{I}_{D}=B$ is a critical case which has to be discussed separately. In principle $\mathcal{D}_{1}$ can have other zeros. But since in general $\bar{I}_{D}$ is changing over time, the direction cannot remain fixed and we can have only a stationary point for $\bar{\delta}$. In this case, if we want a fixed direction over time, i.e. an equilibrium point, we need also a condition on the variation of $\bar{I}_{D}$ : for example a direction $\bar{\delta}_{D}^{*}$ such that $\overline{\bar{I}}_{D}\left(\bar{I}_{D}^{e}, \bar{\delta}_{D}^{*}\right)=0$. We will come back to this point later.

Given the roots and the sign of the function $\mathcal{D}_{1}$, it is straightforward to find the parts of the plane $\left(\bar{I}_{D}, \bar{\delta}\right)$ in which $\dot{\bar{\delta}}$ is positive or negative. The stability of the equilibrium points for $\bar{\delta}$ is given by the sign of the derivative of the function $\dot{\bar{\delta}}=f\left(\bar{I}_{D}, \bar{\delta}, \bar{L}\right)$.

In the first case the equilibrium points are independent of the value of $\bar{I}_{D}$ (and $\left.\bar{L}\right)$, so what is important is the sign of the derivative respect to $\bar{\delta}$ :

$$
\frac{\partial f}{\partial \bar{\delta}}=-\frac{2 a_{2}}{3 \pi} \frac{\overline{P(R)}}{\bar{L}} \cos 2 \bar{\delta} \mathcal{D}_{1}
$$

In the case $\bar{\delta}=\pi / 2$ we have:

$$
\frac{\partial f}{\partial \bar{\delta}}=\frac{2 a_{2}}{3 \pi} \frac{\overline{P(R)}}{\bar{L}} \mathcal{D}_{1}
$$

instead in the cases $\bar{\delta}=0$ and $\bar{\delta}=\pi$ we have:

$$
\frac{\partial f}{\partial \bar{\delta}}=-\frac{2 a_{2}}{3 \pi} \frac{\overline{P(R)}}{\bar{L}} \mathcal{D}_{1}
$$

The stability of these points at a given time depends on the sign of $\mathcal{D}_{1}$, through the shape, the value of $\bar{I}_{D}$ and the sign of the mode $S A M / L A M \pm$. In general, if $\bar{\delta}=\pi / 2$ is stable then $\bar{\delta}=0$ and $\bar{\delta}=\pi$ are unstable and viceversa.

The stability of the equilibrium points in the third case will be discussed in the following. 


\subsection{Equilibrium points for the dynamic inertia $\bar{I}_{D}$}

We are looking for the solutions of the equation

$$
\dot{\bar{I}}_{D}=-\alpha \bar{I}_{D}\left(1-3 \cos ^{2} \bar{\delta}\right) \mathcal{D}_{3}=0 .
$$

We deduce that $\dot{\bar{I}}_{D}=0$ if and only if:

- there is an $\bar{I}_{D}^{d}$ such that $\mathcal{D}_{3}\left(\bar{I}_{D}^{d}\right)=0$, so $\dot{\bar{I}}_{D}=0 \forall \bar{\delta}$

- $\cos ^{2} \bar{\delta}=\frac{1}{3}$

The first case is possible whatever the shape, for $\bar{I}_{D}^{d}=A, \bar{I}_{D}^{d}=C$ and also $\bar{I}_{D}^{d}=B$ because $\lim _{I_{D} \rightarrow B} \mathcal{D}_{3}=0$. So, if we are in principal axis rotation, $\dot{\bar{I}}_{D}=0$ whatever the angular momentum obliquity and we have three equilibrium points for $\bar{I}_{D}$.

In principle, depending on the shape, it is possible to have other zeros of $\mathcal{D}_{3}$, where these are also equilibrium points for $\bar{I}_{D}$.

The second case is when $\bar{\delta}^{*} \cong 54.7^{\circ}$ or $\bar{\delta}^{*} \cong 125.3^{\circ}$. These can be equilibrium points for $\bar{I}_{D}$ only if they are also equilibrium points for $\bar{\delta}$. As we noticed in the previous section, this is possible if $\bar{I}_{D} \equiv \bar{I}_{D}^{e}$, with $\mathcal{D}_{1}\left(\bar{I}_{D}^{e}\right)=0$. In this case we have an equilibrium point $\left(\bar{I}_{D}^{e}, \bar{\delta}^{*}\right)$ for both the direction and the dynamic inertia. Notice that these equilibrium points correspond to the asymptotic states for the obliquity and for the parameter $p=B / I_{D}$ found in [Vokrouhlicky et al. 2007]. The value of the obliquity asymptotic state does not depend on the shape and it is directly comparable with our result, while the exact value of the dynamic inertia asymptotic state depends sensitively on the shape through $\mathcal{D}_{1}$ and it can be qualitatively compared with the numerical examples in section 6 for the given shape models. For example, using the asteroid Toutatis shape model in the $L A M$ case we find a value of $\bar{I}_{D}^{e} / B \cong 0.45$. In the next Figure 6 we show a numerical comparison between the full solution and the secular one at the equilibrium point $\left(\bar{I}_{D}^{e}, \bar{\delta}^{*}\right)$, as described in section 3.7.

Also in this case, once given the roots and the sign of the function $\mathcal{D}_{3}$, it is easy to find the parts of the plane $\left(\bar{I}_{D}, \bar{\delta}\right)$ in which $\dot{\bar{I}}_{D}$ is positive or negative.

\subsubsection{Stability of the equilibrium points for $\bar{I}_{D}$ and $\bar{\delta}$}

The points $\left(\bar{I}_{D}^{d}, 0\right),\left(\bar{I}_{D}^{d}, \pi\right)$ and $\left(\bar{I}_{D}^{d}, \pi / 2\right)$ are equilibrium points for both $\bar{I}_{D}$ and $\bar{\delta}$. Their stability is given by the eigenvalues of the Jacobian matrix $J=\partial(h, f) / \partial\left(\bar{I}_{D}, \bar{\delta}\right)$ computed in the equilibrium points:

$$
J\left(\bar{I}_{D}^{d}, \pi / 2\right)=\left(\begin{array}{cc}
-\alpha \bar{I}_{D}^{d} \mathcal{D}_{3}^{\prime}\left(\bar{I}_{D}^{d}\right) & 0 \\
0 & \alpha \bar{I}_{D}^{d} \mathcal{D}_{1}\left(\bar{I}_{D}^{d}\right)
\end{array}\right),
$$



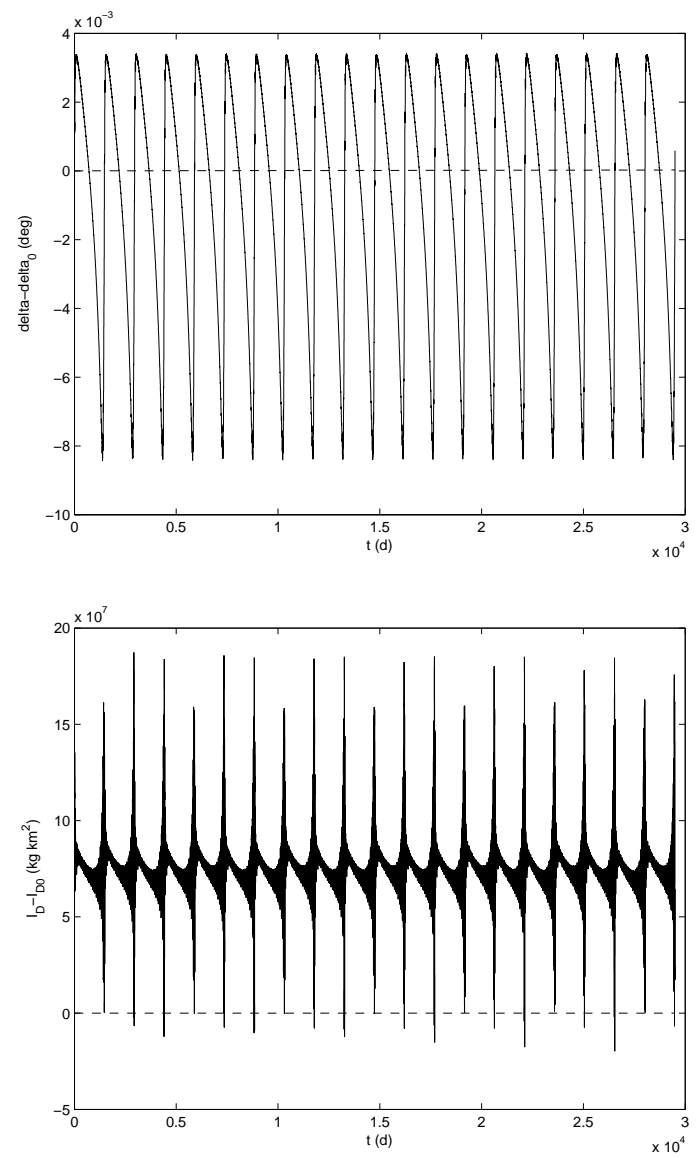

Figure 6: Comparison between the full solution (solid curve) and the averaged solution (dashed line), on the top the obliquity $\delta-\delta_{0}$, on the bottom the dynamic inertia $I_{D}-I_{D 0}$ (initial conditions at the equilibrium $\delta_{0}=\delta^{*} \cong 55^{\circ}$, $\left.I_{D 0}=\bar{I}_{D}^{e}\right)$, both plotted over a $\Delta t \cong 20 T_{\text {orb }}$ time span. 
then $\left(\bar{I}_{D}^{d}, \pi / 2\right)$ can be a saddle or a node, depending on the sign of $\mathcal{D}_{3}^{\prime}\left(\bar{I}_{D}^{d}\right)$ and $\mathcal{D}_{1}\left(\bar{I}_{D}^{d}\right)$.

And:

$$
J\left(\bar{I}_{D}^{d}, 0\right)=J\left(\bar{I}_{D}^{d}, \pi\right)=\left(\begin{array}{cc}
2 \alpha \bar{I}_{D}^{d} \mathcal{D}_{3}^{\prime}\left(\bar{I}_{D}^{d}\right) & 0 \\
0 & -\alpha \bar{I}_{D}^{d} \mathcal{D}_{1}\left(\bar{I}_{D}^{d}\right)
\end{array}\right),
$$

then $\left(\bar{I}_{D}^{d}, 0\right)$ and $\left(\bar{I}_{D}^{d}, \pi\right)$ are both saddles or both nodes. In particular they are saddles if $\left(\bar{I}_{D}^{d}, \pi / 2\right)$ is a saddle, and nodes if $\left(\bar{I}_{D}^{d}, \pi / 2\right)$ is a node (with opposite stability). We found the same result in the case of principal axis rotation $\bar{I}_{D} \equiv C$ (example in [Scheeres \& Mirrahimi 2008]), where the equilibrium points for the obliquity are $0, \pi$ and $\pi / 2$ with opposite stability.

The point $\left(\bar{I}_{D}^{e}, \bar{\delta}_{D}^{*}\right)$ that we found above is also an equilibrium point for both $\bar{I}_{D}$ and $\bar{\delta}$. In this case:

$$
J\left(\bar{I}_{D}^{e}, \bar{\delta}_{D}^{*}\right)=\left(\begin{array}{cc}
0 & -3 \alpha \bar{I}_{D}^{e} \sin 2 \bar{\delta}^{*} \mathcal{D}_{3}\left(\bar{I}_{D}^{e}\right) \\
-\frac{\alpha}{2} \sin 2 \bar{\delta}^{*} \mathcal{D}_{1}^{\prime}\left(\bar{I}_{D}^{e}\right) & 0
\end{array}\right) .
$$

The eigenvalues of this matrix are:

$$
\lambda_{1,2}= \pm \sqrt{\frac{16}{3} \alpha^{2} \bar{I}_{D}^{e} \mathcal{D}_{1}^{\prime}\left(\bar{I}_{D}^{e}\right) \mathcal{D}_{3}\left(\bar{I}_{D}^{e}\right)}
$$

Thus

- if $\mathcal{D}_{1}^{\prime}\left(\bar{I}_{D}^{e}\right) \mathcal{D}_{3}\left(\bar{I}_{D}^{e}\right)>0$ we have a hyperbolic point, in particular a saddle;

- if $\mathcal{D}_{1}^{\prime}\left(\bar{I}_{D}^{e}\right) \mathcal{D}_{3}\left(\bar{I}_{D}^{e}\right)<0$ we have a nonhyperbolic point, in particular a center;

- if $\mathcal{D}_{1}^{\prime}\left(\bar{I}_{D}^{e}\right) \mathcal{D}_{3}\left(\bar{I}_{D}^{e}\right)=0$ we have a nonhyperbolic point with two zero eigenvalues.

Notice that if $\mathcal{D}_{1}$ and $\mathcal{D}_{3}$ have only one root in the open interval $(A, B)$ (or $(B, C)$ ), respectively $\bar{I}_{D}^{e}$ and $\bar{I}_{D}^{d}$, then the function $\mathcal{D}_{1} \mathcal{D}_{3}$ has exactly two roots, and the signs of $\mathcal{D}_{1}^{\prime}\left(\bar{I}_{D}^{e}\right) \mathcal{D}_{3}\left(\bar{I}_{D}^{e}\right)$ and $\mathcal{D}_{1}\left(\bar{I}_{D}^{d}\right) \mathcal{D}_{3}^{\prime}\left(\bar{I}_{D}^{d}\right)$ must be opposite. Thus if the equilibrium points $\left(\bar{I}_{D}^{e}, \bar{\delta}^{*}\right)$ are centers the equilibrium points $\left(\bar{I}_{D}^{d}, \bar{\delta}\right)$ for $\bar{\delta}=0, \pi / 2, \pi$ are saddles.

A detailed analysis of the critical case $\mathcal{D}_{1}^{\prime}\left(\bar{I}_{D}^{e}\right) \mathcal{D}_{3}\left(\bar{I}_{D}^{e}\right)=0$ is beyond the scope of this work. We notice that if $\mathcal{D}_{3}\left(\bar{I}_{D}^{e}\right)=0$, then the points $\left(\bar{I}_{D}^{e}, \bar{\delta}\right)$ are all equilibrium points for both $\bar{I}_{D}$ and $\bar{\delta}$. 


\subsection{Equilibrium points for the angular momentum magni- tude}

The equilibrium and stationary points for $\bar{L}$ are given by the equation

$$
\dot{\bar{L}}=\frac{a_{2} \overline{P(R)}}{3 \pi}\left(1-3 \cos ^{2} \bar{\delta}\right) \mathcal{D}_{1}=0 .
$$

We deduce that $\dot{\bar{L}}=0$ if and only if:

- there is an $\bar{I}_{D}^{l}$ such that $\mathcal{D}_{1}\left(\bar{I}_{D}^{l}\right)=0$, so $\dot{\bar{L}}=0 \forall \bar{\delta}$

$$
\text { - } \cos ^{2} \bar{\delta}=\frac{1}{3}
$$

In both cases, if $\bar{I}_{D}^{l} \neq B$, to be equilibrium points for $\bar{L}$ we need them to also be equilibrium points for $\bar{I}_{D}$ and $\bar{\delta}$. From the previous sections we know that these are the cases for $\bar{I}_{D}=\bar{I}_{D}^{e}$ with $\mathcal{D}_{1}\left(\bar{I}_{D}^{e}\right)=0, \bar{\delta}^{*}=\arccos (1 / \sqrt{3}) \cong 54.7^{\circ}$ and $\bar{\delta}^{*} \cong 125.3^{\circ}$. Figure 7 shows a numerical comparison between the full and the secular solutions for $L$, when the initial conditions are chosen at the equilibrium $\left(\bar{I}_{D}^{e}, \bar{\delta}^{*}\right)$ (see section 4.2 ). Notice that these correspond to the zero-torque obliquities found in [Nesvorny \& Vokrouhlicky 2007]. The stability of these points has already been discussed, because it does not depend on the value of $\bar{L}$. The parts of the plane $\left(\bar{I}_{D}, \bar{\delta}\right)$ in which $\dot{\bar{L}}$ is positive or negative are easily obtainable.

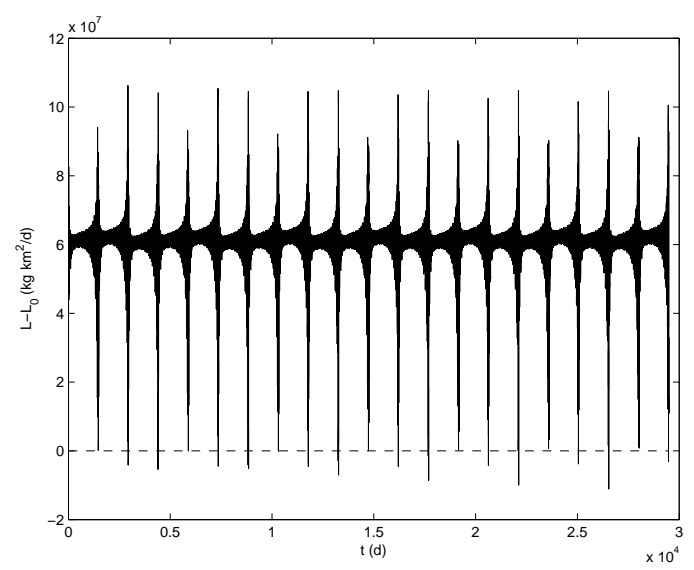

Figure 7: Comparison between the full solution (solid curve) and the averaged solution (dashed line) for the angular momentum $L-L_{0}$ (initial conditions at the equilibrium $\delta_{0}=\delta^{*} \cong 55^{\circ}, I_{D 0}=\bar{I}_{D}^{e}$ ), plotted over a $\Delta t \cong 20 T_{\text {orb }}$ time span. 
Note. We have seen that in the case of $\bar{I}_{D}=B$ we have always $\dot{\bar{\delta}}=\dot{\bar{L}}=\dot{\bar{I}}_{D}=0$, then this is an equilibrium point for all the three fundamental quantities. However this is only true for the averaged equations, not for the full dynamical system. We will see that this is a critical situation which has to be treated separately (see the section 5 "Transition and chaotic behaviour").

\subsection{Generic examples of dynamics in the plane $\left(\bar{I}_{D}, \bar{\delta}\right)$}

Here we present two generic examples of the dynamics flow in the plane $\left(\bar{I}_{D}, \bar{\delta}\right)$. In the first example we suppose that the functions $\mathcal{D}_{1}$ and $\mathcal{D}_{3}$ have exactly one root in the open interval $(A, B)$ and one root in the open interval $(B, C)$. We indicate with $\bar{I}_{D}^{e}$ the roots of $\mathcal{D}_{1}$ and with $\bar{I}_{D}^{d}$ the roots of $\mathcal{D}_{3}$ in the open intervals $(A, B)$ and $(B, C)$.

In the second example we suppose that $\mathcal{D}_{1}$ and $\mathcal{D}_{3}$ do not have roots in these intervals.

The structure of the orbits is obtained from the level curves of the first integral function $F$ in formula (21). The direction of the orbits is given by the sign \pm of the mode $S A M_{ \pm}$or $L A M_{ \pm}$: changing it has the only effect of changing the direction of the motion. Since it is possible for the $\mathcal{D}_{k}$ functions to have roots in the $L A M$ case and to have no roots in the $S A M$ case, or viceversa, mixed phase diagrams are possible (see also section 6 ).

In the first example (Figure 8) we suppose that $\mathcal{D}_{1}^{\prime}\left(\bar{I}_{D}^{e}\right) \mathcal{D}_{3}\left(\bar{I}_{D}^{e}\right)<0$ for both the zeros of $\mathcal{D}_{1}$ in $(A, B)$ and in $(B, C)$, i.e., non-hyperbolic equilibrium points. And we also suppose that the roots of $\mathcal{D}_{3}$ are closer to the value $B$ with respect to the roots of $\mathcal{D}_{1}$.

In the second example (Figure 9 ) we suppose that $\mathcal{D}_{1}$ and $\mathcal{D}_{3}$ do not have roots in the open intervals $(A, B)$ and $(B, C)$. We suppose also that $\mathcal{D}_{1}\left(\bar{I}_{D}\right) \mathcal{D}_{3}^{\prime}\left(\bar{I}_{D}\right)>0$ for $\bar{I}_{D}=A, C$. The behaviour of the orbits approaching the value $\bar{I}_{D}=B$ needs to be treated separately (see section 5 ). 


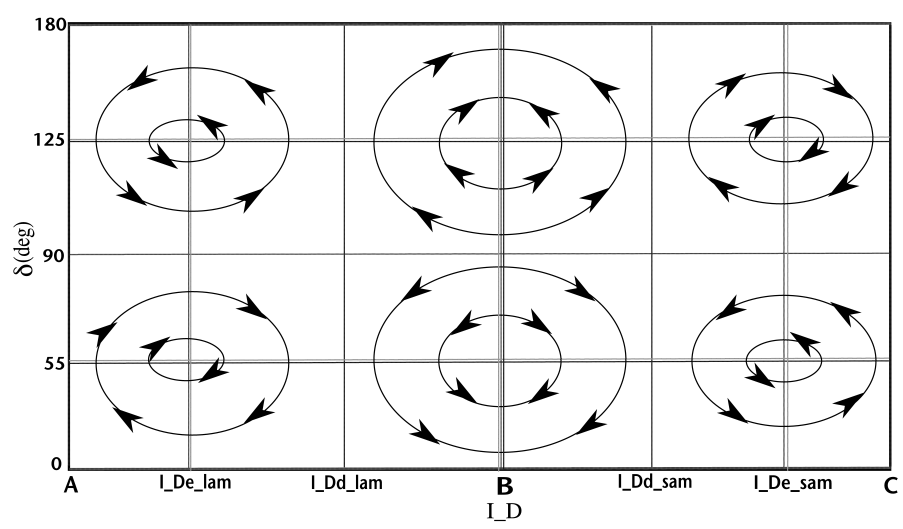

Figure 8: In the plane $\left(\bar{I}_{D}, \bar{\delta}\right)$, the vertical lines at $A, C$ and $\bar{I}_{D}^{d}$ are stationary points for $\bar{I}_{D}$, the ones at $\bar{I}_{D}^{e}$ are stationary points for $\bar{\delta}$ and $\bar{L}$. The horizontal lines at $\sim 55^{\circ}$ and $\sim 125^{\circ}$ are stationary points for $\bar{I}_{D}$ and $\bar{L}$, the ones at $0^{\circ}, 90^{\circ}$ and $180^{\circ}$ are stationary points for $\bar{\delta}$. The value $B$ is a critical value (section 5). In this case the orbits are closed around the center equilibrium points $\left(\bar{I}_{D}^{e}, \bar{\delta}^{*} \sim 55^{\circ}\right)$ and $\left(\bar{I}_{D}^{e}, \bar{\delta}^{*} \sim 125^{\circ}\right)$. The points $\left(\bar{I}_{D}^{d}, 0\right),\left(\bar{I}_{D}^{d}, \pi\right)$ and $\left(\bar{I}_{D}^{d}, \pi / 2\right)$ are saddles.

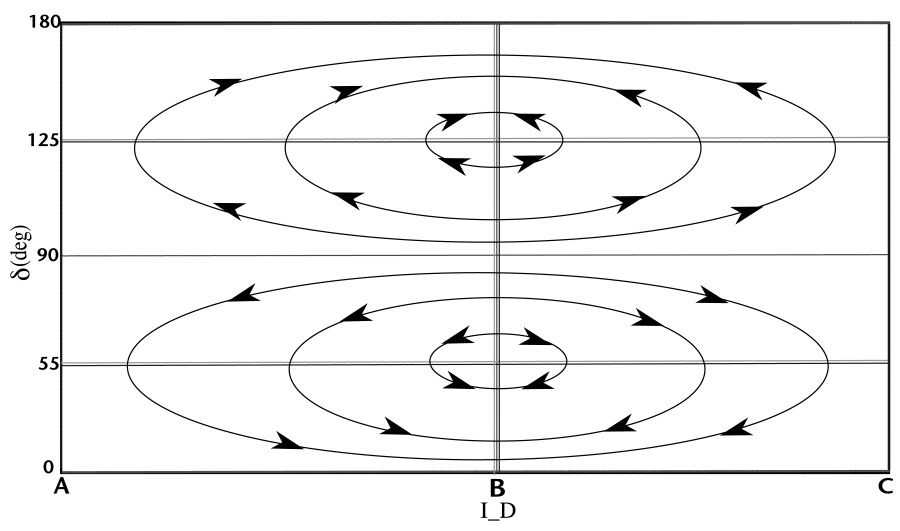

Figure 9: In the plane $\left(\bar{I}_{D}, \bar{\delta}\right)$, the vertical lines at $A$ and $C$ are stationary points for $\bar{I}_{D}$. The horizontal lines at $\sim 55^{\circ}$ and $\sim 125^{\circ}$ are stationary points for $\bar{I}_{D}$ and $\bar{L}$, the ones at $0^{\circ}, 90^{\circ}$ and $180^{\circ}$ are stationary points for $\bar{\delta}$. The value $B$ is a critical value (section 5 ). The equilibrium points $\left(\bar{I}_{D}, 0\right)$, $\left(\bar{I}_{D}, \pi\right)$ and $\left(\bar{I}_{D}, \pi / 2\right)$ for $\bar{I}_{D}=A, C$ are saddles. 
In principle other behaviours are possible, for example we can consider the case $\mathcal{D}_{1}^{\prime}\left(\bar{I}_{D}^{e}\right) \mathcal{D}_{3}\left(\bar{I}_{D}^{e}\right)>0$ for both the zeros of $\mathcal{D}_{1}$ in $(A, B)$ and in $(B, C)$, i.e., hyperbolic equilibrium points. Or cases in which the roots of $\mathcal{D}_{1}$ are closer to the value $B$ with respect to the roots of $\mathcal{D}_{3}$. We could also consider hypothetical cases in which the function $\mathcal{D}_{1}$ has roots and the function $\mathcal{D}_{3}$ does not. However, the qualitative behaviours described in this section are the only ones that we found from all the asteroid shape models that we tested (see section 6).

Confronting the plot in Figure 8 with the corresponding ones in [Vokrouhlicky et al. 2007] we can notice that the former contains closed cycles with no way to tend towards the equilibrium point, while in the latters there are cases in which the solution tends asymptotically to the equilibrium. This is probably the principal difference between the present results and the numerical simulations of [Vokrouhlicky et al. 2007]. Possible reasons for these differences are discussed in the next subsection 4.5.

\subsection{Generalizations}

In section 2.4, while modelling the YORP torque, we pointed out two important generalizations that we made to the approximations:

- considering a better approximation of the illumination function $f$, up to higher order terms (e.g. to $\left.(\mathbf{n} \cdot \mathbf{u})^{4}\right)$, and considering the selfshadowing phenomena of the body;

- considering the dissipation of energy.

In section 3.5 we noticed that a better approximation of $f$ involves the computation of a large number of terms, and we are not considering the possibility of doing this computation here. In the following we discuss qualitatively, and not rigorously, some possible consequences of these approximations.

\subsubsection{The illumination function}

Considering additional terms in the series development of the illumination function acts as a perturbation on the dynamics previously described:

$$
\left\{\begin{aligned}
\dot{\bar{I}}_{D} & =h\left(\bar{I}_{D}, \bar{\delta}, \bar{L}\right)+\frac{1}{\bar{L}} \epsilon_{1}\left(\bar{I}_{D}, \bar{\delta}\right) \\
\dot{\bar{\delta}} & =f\left(\bar{I}_{D}, \bar{\delta}, \bar{L}\right)+\frac{1}{\bar{L}} \epsilon_{2}\left(\bar{I}_{D}, \bar{\delta}\right) \\
\dot{\bar{L}} & =g\left(\bar{I}_{D}, \bar{\delta}\right)+\epsilon_{3}\left(\bar{I}_{D}, \bar{\delta}\right) .
\end{aligned}\right.
$$

However, it is not obvious how much the effect of such a perturbation would be for different shapes, it could be stronger for shapes with possible selfshadowing. 
We can only conjecture that the dynamics does not change much in the case of the hyperbolic equilibrium points, because the linear part of the system should not be perturbed enough to change its kind.

Instead, in the case of the non-hyperbolic equilibrium points, we could expect them to become hyperbolic, and to have a deviation from the closed orbits (the ones along the first integral level curves), moving on a spiral toward the equilibrium points, or escaping from them (the center becomes a focus). A qualitative example of this effect is given in Figure 10.
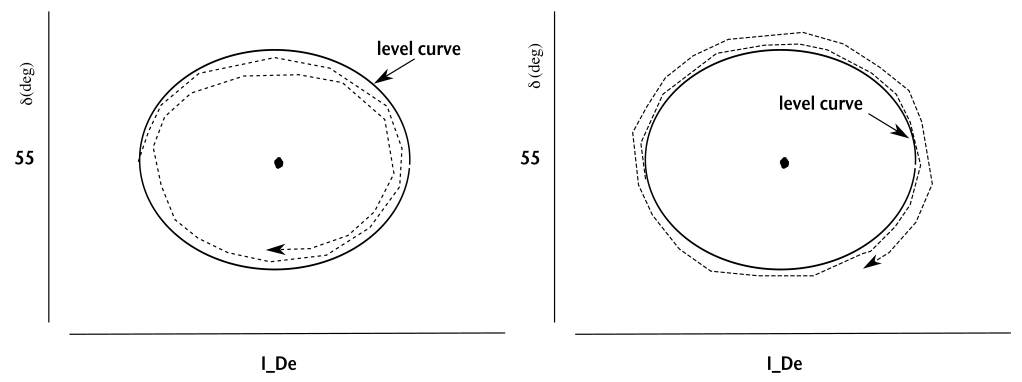

Figure 10: A qualitative example (conjectured) of the solution deviating from the level curve of the first integral. Going toward the equilibrium point (left), or escaping from it (right).

As we anticipated, the numerical simulations in [Vokrouhlicky et al. 2007] actually show asymptotic states and a more complex dynamics. Although the asymptotic states clearly correspond to the equilibrium points $\left(\bar{I}_{D}^{e}, \bar{\delta}^{*}\right)$ that we also found, in those simulations there seems to be no trace of the roots of the function $\mathcal{D}_{3}$. Moreover they found that when the solution is close to the asymptotic state the angular momentum magnitude rate $\dot{L}$ is constant. It is possible that these differences are due to the fact that they also included gravitational torques due to the Sun and to the different model they used for the illumination function, which is more realistic in the numerical simulations and it can easily destroy the integrability of our simplified model. It is worth to notice that in [Statler 2009] it is shown, using full numerical simulations, the sensitivity of the YORP effect to small changes in the shape of the body.

A better model for $f$ should be necessary to describe the averaged behaviour of the rotational dynamics under the YORP torque in a more accurate way.

\subsubsection{The dissipation of energy}

The dissipation of energy, discussed in sections 2.5 and 3.6, involves the angular momentum magnitude as an important parameter. Indeed, if $L$ is small the effect of the dissipation is small, and in this case we have a 
small perturbation of the dynamics with no dissipation. Again, the effect would not be important in the case of hyperbolic equilibrium points, while it could drive the motion toward the equilibrium points (on a spiral) in the case of the non-hyperbolic equilibrium points. An interesting result here is that, for moderate values of $L$, we may have non principal axis states as an asymptotic state even with energy dissipation. Instead, when $L$ is large, dissipation tends to dominate the YORP effect. In this case we would not have equilibrium points for $\bar{I}_{D}$ anymore, and the result will be that the motion tends to the principal $S A M$ axis rotation $\left(\bar{I}_{D} \rightarrow C\right)$.

\section{Transition and chaotic behaviour}

In this section we describe qualitatively the behaviour of the solution when it approaches the critical value $\bar{I}_{D}=B$.

Since the functions $\mathcal{D}_{k}^{ \pm}\left(\bar{I}_{D}\right)$ change sign when we change the sign mode \pm , the phase diagram changes behaviour after every transition $S A M \leftrightarrow L A M$, depending on which of the four following transitions happen:

$S A M_{+} \leftrightarrow L A M_{+}, S A M_{+} \leftrightarrow L A M_{-}, S A M_{-} \leftrightarrow L A M_{+}, S A M_{-} \leftrightarrow L A M_{-}$

Note that these are the only possible transitions, since it is not possible to have a transition $S A M_{+} \leftrightarrow S A M_{-}$or $L A M_{+} \leftrightarrow L A M_{-}$.

So, as long as the rotation state stays in the same mode, for a long enough time span to perform an averaged solution, then, starting from some initial conditions $\left(\bar{I}_{D 0}, \bar{\delta}_{0}\right)$ it is possible to perform a qualitative study of the averaged solution $\left(\bar{I}_{D}(t), \bar{\delta}(t)\right)$. This gives us information on the nonprincipal axis rotation, on the direction of the angular momentum, and

also on the sign of $\dot{\bar{L}}(t)$, which gives us information on the acceleration or deceleration of the rotation state of the body.

However, what happens when we approach the transition point $\bar{I}_{D}=B$ is a delicate matter, because we cannot know a priori if the system is going to make a transition to $\mathrm{a}+$ or a - mode, which depends on the rotation state in the body fixed frame. In particular, the sign of the mode is given by the angles $\theta$ and $\psi$, so we should go back to the full perturbed solution to know what these angles are doing near the transition.

When the full solution is close to the transition point we find it has a chaotic behaviour: the system can transit from a $S A M_{ \pm}$to a $L A M_{ \pm}$ several times before reaching a stable condition, at which point it makes sense to continue the averaged study. We will explain this with the following example. 
Example. To better understand why this happens and in what sense the transition has a chaotic behaviour, we recall that the direct formula (2) for $I_{D}$ depends on the angles $\theta$ and $\psi$, the unperturbed behaviour of these angles is:

- $\left.S A M_{+}: \theta \in\right] 0, \frac{\pi}{2}[$ oscill., $\psi \in[0,2 \pi]$ circul.

- $\left.S A M_{-}: \theta \in\right] \frac{\pi}{2}, \pi[$ oscill., $\quad \psi \in[0,2 \pi]$ circul.

- $\left.L A M_{+}: \theta \in\right] 0, \pi\left[\right.$ libr. around $\left.\frac{\pi}{2}, \quad \psi \in\right] 0, \pi\left[\right.$ libr. around $\frac{\pi}{2}$

- $\left.L A M_{-}: \theta \in\right] 0, \pi\left[\right.$ libr. around $\left.\frac{\pi}{2}, \quad \psi \in\right] \pi, 2 \pi\left[\right.$ libr. around $\frac{\pi}{2}$

and the period of $\psi$ is exactly twice the period of $\theta$.

We can examine as an example a transition $S A M_{+} \rightarrow L A M$. Let us suppose the body is in a $S A M_{+}$state and we are approaching a transition, i.e. $I_{D} \sim B$. This means that the angle $\theta$ is approaching the value $\pi / 2$, while the angle $\psi$ is still circulating in $[0,2 \pi]$. Because $\psi$ is circulating with twice the period of $\theta$, immediately after $I_{D}$ transitions to $<B$, the angle $\psi$ has "the same probability" to be in the interval $] 0, \pi[$ or $] \pi, 2 \pi[$. In the first case the body is captured in a $L A M_{+}$state, in the second case in a $L A M_{-}$state. A qualitative example of the level curves of the function $I_{D}=I_{D}(\theta, \psi)$ on the torus $\theta, \psi$ and examples of unperturbed orbits and possible perturbed transitions are given in the next Figure 11.

After the transition, the solution can either stay close to the value $I_{D} \sim$ $B$, and we can have a transition again, or instead, depending on the sign of $\dot{\bar{I}}_{D}$ (see subsection 5.2), it can go far enough from the transition zone to be captured in a definite mode for a longer time span.

Def. We say the the system is in a steady state condition if $\bar{I}_{D}$ remains far from the critical point $\bar{I}_{D}=B$ for a long time span, where long is measured with respect to the orbital period $T_{\text {orb }}$. 


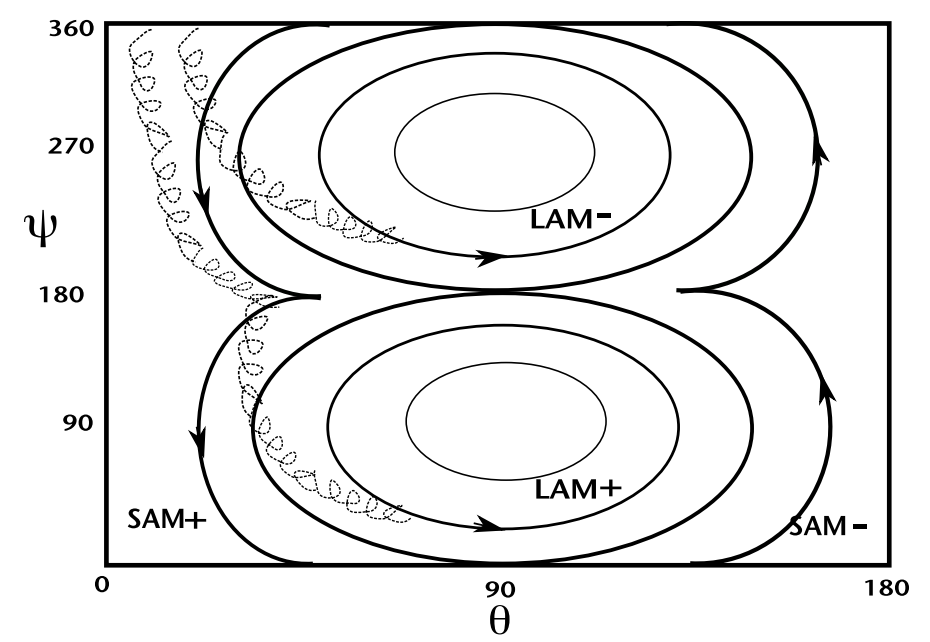

Figure 11: Qualitative cartoon of the level curves of the function $I_{D}=$ $I_{D}(\theta, \psi)$ on the torus $\theta, \psi$ (solid lines), the different modes $S A M$ and $L A M$ are highlighted. The dashed curves are examples of perturbed orbits which can transition from the $S A M_{+}$mode to the $L A M_{-}$or to the $L A M_{+}$mode.

\subsection{The transition probability}

In order to predict what kind of transition the system is going to have, using only the averaged equations, we define a transition probability:

Def1. Assume that $\bar{I}_{D}$ is monotonically approaching the critical value $\bar{I}_{D}=B$. We define the transition probability $P_{t}^{ \pm}$to the other mode ( $S A M$ or $L A M)+$ or - , as the percentage of the initial conditions $\theta_{0}, \phi_{0}, \psi_{0}$ such that the body makes a transition to the mode + or the mode - .

According to the previous discussion, we assume, as a rule, that the transition probability to the other mode + or - is $P_{t}^{+}=P_{t}^{-}=0.5$.

Def2. Once a transition occurs, we define in the same way the probability to have another transition, $P_{n t}$, before reaching a steady state condition.

In general we can have two kinds of situations near the critical value $\bar{I}_{D}=B$ :

- The potential transition modes:

$$
S A M \quad \text { with } \quad \dot{\bar{I}}_{D}<0 \quad \text { or } \quad L A M \quad \text { with } \quad \dot{\bar{I}}_{D}>0
$$

- The potential steady state modes:

$$
S A M \quad \text { with } \quad \dot{\bar{I}}_{D}>0 \quad \text { or } \quad L A M \quad \text { with } \quad \dot{\bar{I}}_{D}<0
$$


We assume that the probability to have another transition whenever we are in a "potential transition" mode is $P_{n t}^{a}=1$. We note that, for a non-averaged dynamical system, that this probability could be less than 1 .

We assume that the probability to have another transition when we are in a potential steady state mode is $P_{n t}^{s}=0.5$, but this is not an intuitive rule as the ones above, and it is only a first guess. While intuitively one would think this probability should be " 0 ", our numerical results show that the short period fluctuations about the secular motion allow for a non-zero probability here.

As a consequence, assuming that following a transition we enter to a potential transition mode with probability $P_{t}^{a}$ and enter a potential steady state mode with probability $P_{t}^{s}$, the total probability to have another transition is

$$
P_{n t}=P_{t}^{a} P_{n t}^{a}+P_{t}^{s} P_{n t}^{s}
$$

For example, assuming that one mode (e.g. the + ) is a transition approaching and the other (the - ) is a potentially steady state, with our previous assumptions we have $P_{n t}=0.75$.

\subsection{Chaotic phase and steady state evolution}

As anticipated before, when we are approaching a transition we can have only the two following behaviours:

- The system stays for a while in a chaotic state, undergoing several transitions: the average behaviour is basically $\bar{I}_{D} \cong B, \dot{\bar{\delta}} \cong 0$ and $\dot{\bar{L}} \cong 0$.

- The system falls into a steady state mode and it goes far from the transition point $\bar{I}_{D}=B$.

Once we know what is the probability for the system to have a new transition before reaching a steady state condition, we can compute the probability to reach a steady state condition after $N$ transitions. This provides us with an idea of the time span in which the system persist in chaotic behaviour.

For example, with our previous hypothesis, the probability to have at least $N=10$ subsequent transitions is $P=\left(P_{n t}\right)^{N} \cong 0.05$, which is low. Then the probability to reach a steady state condition after more than $N=$ 10 subsequent transitions is $P^{s} \cong 0.95$.

If we assume that there is, on average, one or two transitions per year, this means that the chaotic behaviour will last from 5 to 10 years. 
Finally, if we come back to the situation in which the system is approaching the transition for the first time, we have two possible steady state conditions that can happen after a chaotic phase, the probability to get to each one of them is 0.5 .

\subsection{Tests on the chaotic transition}

In order to verify numerically the previous discussion about the chaotic transition of a body under torque, we used the asteroid Toutatis shape model to perform some tests.

In this case the transition modes are $S A M_{+}$and $L A M_{+}$, while the steady state modes are $S A M_{-}$and $L A M_{-}$(see section 6.1). Using suitable initial conditions, we obtain an averaged solution which is approaching the critical value $\bar{I}_{D}=B$. In these tests we are approaching the transition from the $S A M_{+}$mode.

To see what happens during the transition, we start a full numerical integration close to the critical value, choosing the initial conditions for the angles $\theta, \phi$ and $\psi$ in a suitable way to have the right initial value for $I_{D}$. This choice is not unique, and we find that choosing slightly different values for these initial conditions yields very different evolutions.

In the first example the body ultimately transitions from $S A M_{+}$to $L A M_{-}$, and during the chaotic phase it undergoes the following transitions before reaching a steady state condition:

$$
\begin{aligned}
& S A M_{+} \rightarrow L A M_{+} \rightarrow S A M_{-} \rightarrow L A M_{-} \rightarrow S A M_{+} \rightarrow \\
& \rightarrow L A M_{-} \rightarrow S A M_{-} \rightarrow L A M_{-} \rightarrow S A M_{+} \rightarrow L A M_{-} .
\end{aligned}
$$



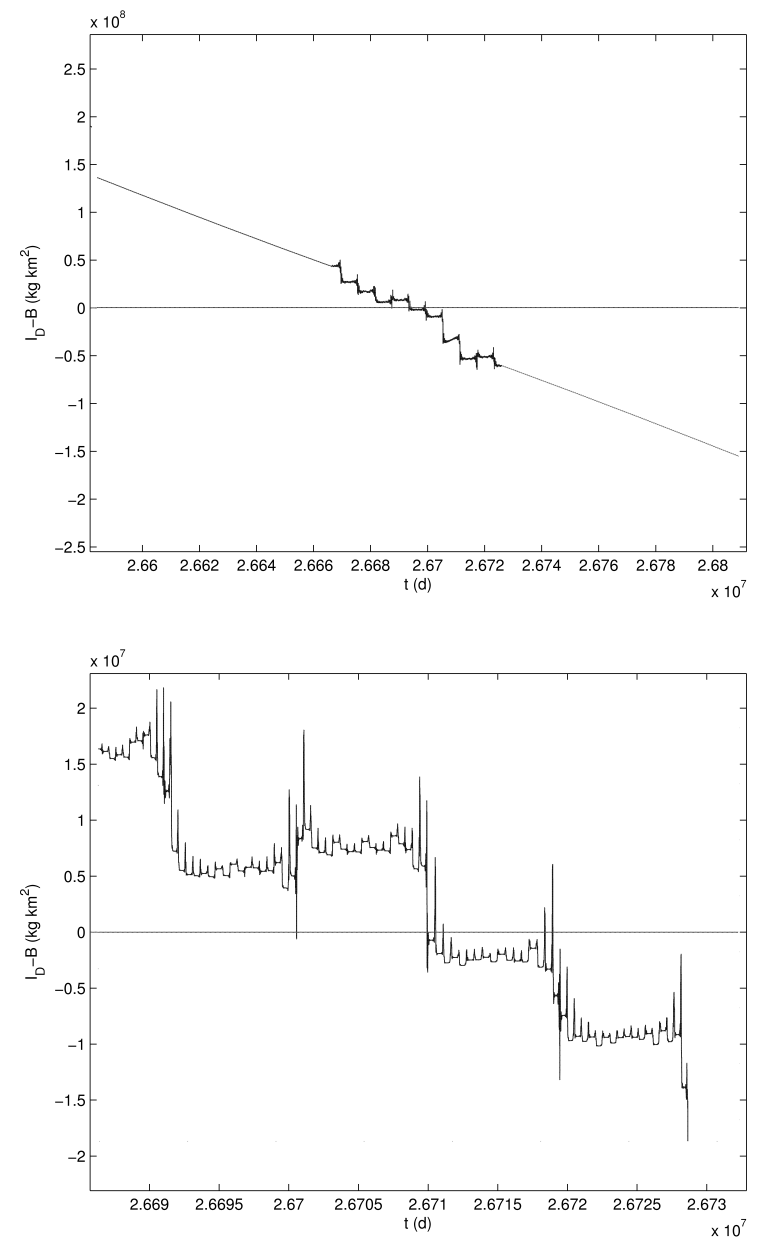

Figure 12: Time versus the dynamic inertia $I_{D}$. On the top, the full numerical simulation going through the transition point $I_{D}=B$ (horizontal line), connected with two branches of the secular solution. On the bottom a detail of the transitions. In this case the system goes from the $S A M_{+}$mode to the $L A M_{-}$mode. 
In the second example, we change the initial conditions a small amount with respect to the first example, and the body has instead a transition from $S A M_{+}$to $S A M_{-}$, and during the chaotic phase it undergoes the following transitions before reaching a steady state condition:

$$
S A M_{+} \rightarrow L A M_{+} \rightarrow S A M_{-} .
$$
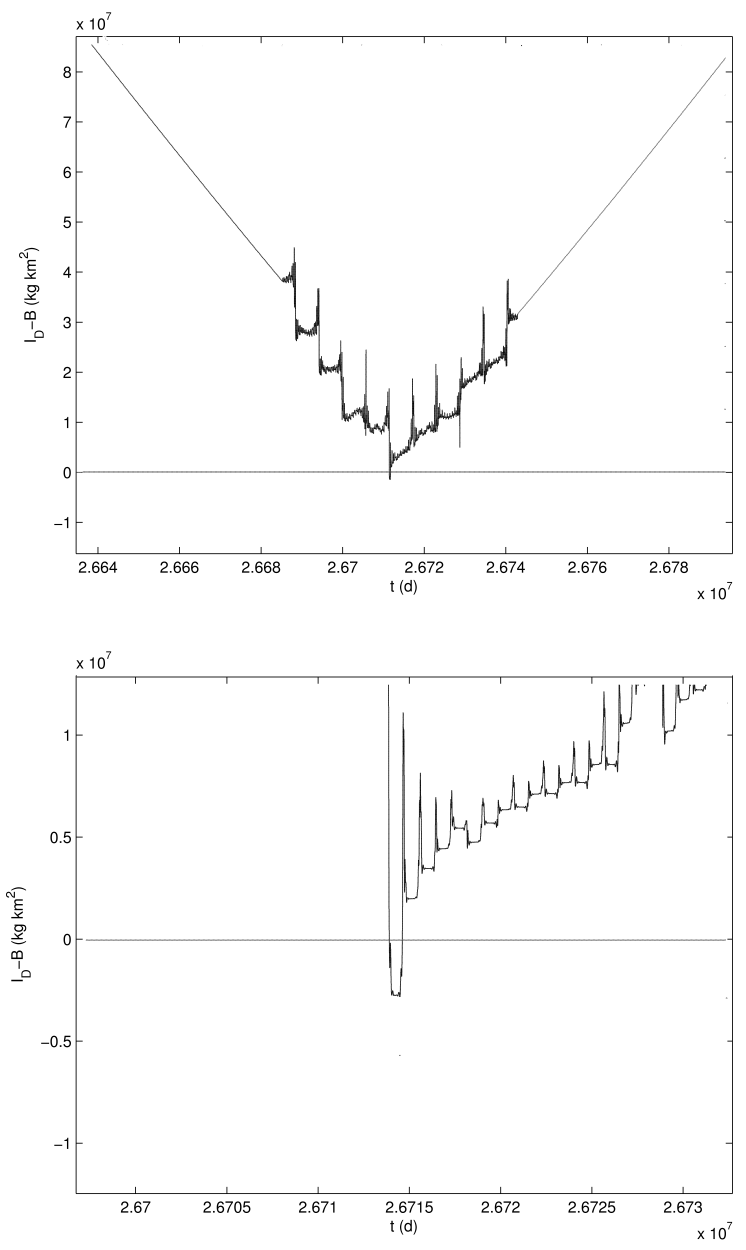

Figure 13: Time versus the dynamic inertia $I_{D}$. On the top, the full numerical simulation going through the transition point $I_{D}=B$ (horizontal line), connected with two branches of the secular solution. On the bottom a detail of the transitions. In this case the system goes from the $S A M_{+}$mode to the $S A M_{-}$mode. 


\subsection{Other possible transitions}

Another effect similar to the transition phenomenon described in this section, is the behaviour of the solution close to the stationary points $\dot{\bar{I}}_{D}=0$ and $\dot{\bar{\delta}}=0$. Since they are stationary points for the averaged dynamical system $(20)$, the secular solution cannot cross them in the plane $\left(\bar{I}_{D}, \bar{\delta}\right)$. However, if the secular solution is close enough to these lines, then, because the true solution has non zero finite amplitude around the secular one, it can eventually cross the stationary point, and there is a non zero probability for the solution to fall into the other side with respect to the lines $\dot{\bar{I}}_{D}=0$ or $\dot{\bar{\delta}}=0$. According to this mechanism, it is possible for the true solution to go through the whole plane $\left(\bar{I}_{D}, \bar{\delta}\right)$ regardless of the presence of stationary points for the averaged solution. Indeed, the true solution can remain close to the stationary value for a long time span, following, on average, orbits on both sides of that value. But since the secular orbits have to go through the level curves of the first integral (21), at some point the solution has to go far away from the stationary value. On which side of the stationary value the true solution goes at the end is a matter of probability. An idea of this behaviour is given in the next Figure 14, obtained using the asteroid Toutatis shape model (see section 6).

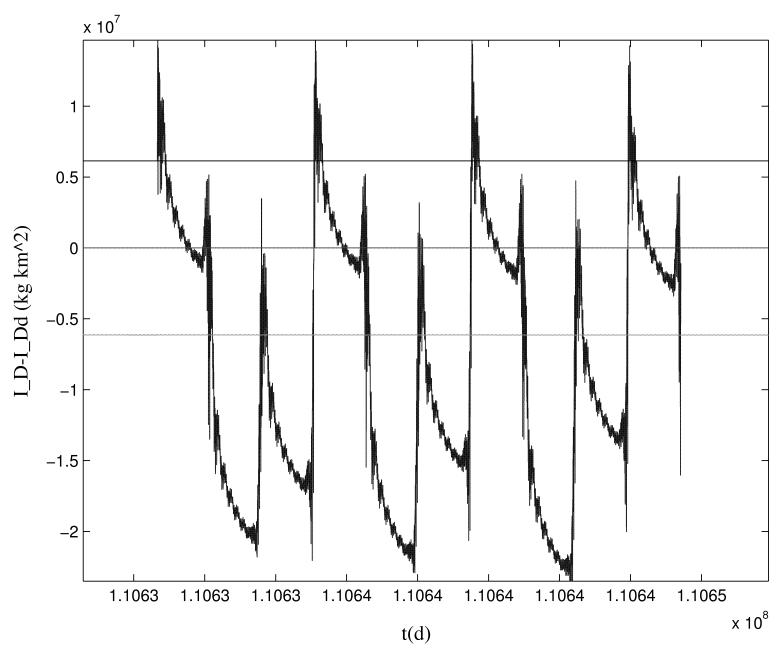

Figure 14: On the vertical axis the dynamic inertia $I_{D}$, translated by the stationary value $\bar{I}_{D}^{d}$. On the horizontal axis the time $\left(\Delta t \cong 10 T_{\text {orb }}\right)$. The solid and the dashed horizontal lines are the secular solutions close to the stationary value (zero horizontal line). The true solution oscillates going through both sides with respect to the stationary point. 


\section{Tests on asteroids}

In the sections $3.6,3.7,5.3$ and 5.4 we showed the results from numerical integrations in which we used the asteroid Toutatis shape model. The model we used consists in $N \cong 3000$ triangular facets. In this section we test seven different asteroid shape models, all of them consisting in thousands of triangular facets. The asteroids we test are Toutatis, 1998 KY26, Castalia, Golevka (shape models from [Neese 2004]), the asteroid 1992 SK, from [Busch et al 2006], the asteroid Nereus, from [Brozovic et al. 2009] and the asteroid $1999 \mathrm{KW}_{4}$, from [Ostro et al 2006].

\subsection{Computation of the $\mathcal{D}_{k}$ functions}

We compute here the $\mathcal{D}_{k}$ functions for all of them, using a discretization of the interval $(A, C)$ with $\sim 100000$ points. The functions are normalized using the mean radius cube $R_{m}^{3}$ of each asteroid, and they are plotted together in the next Figures 17 - 20, assuming the positive sign for the modes $S A M$ and $L A M$. We recall that $\mathcal{D}_{k}^{-}=-\mathcal{D}_{k}^{+}$. Finally, we summarize the main features of these functions in Tables 1 and 2 , in light of the discussion of section 4 .

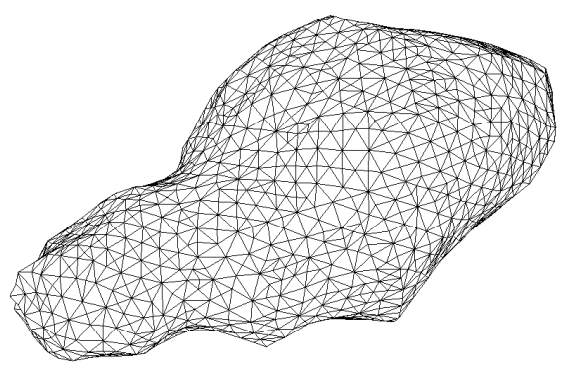

Figure 15: The asteroid Toutatis shape model with $N \cong 3000$ facets. 

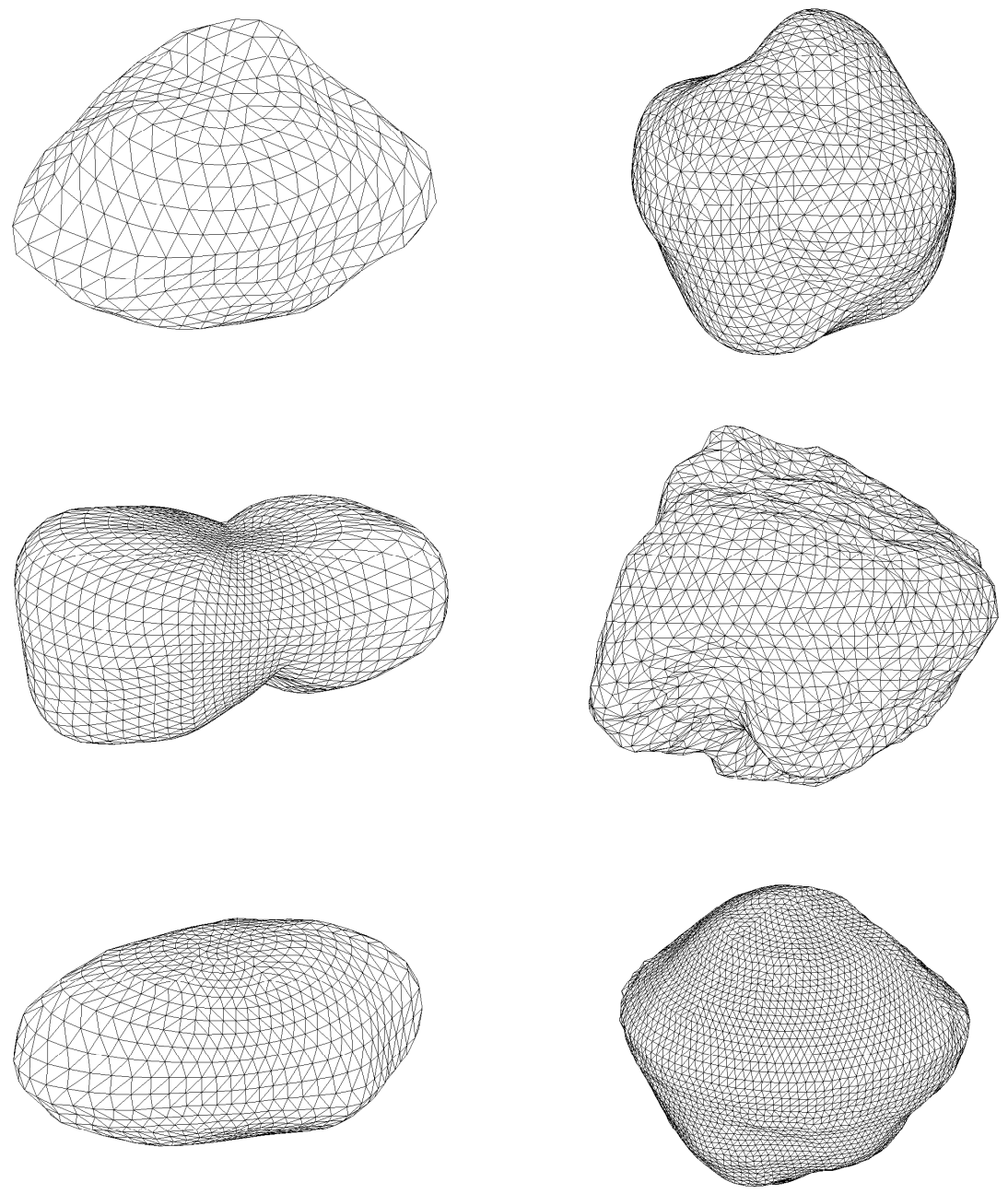

Figure 16: From the top on the left to the bottom on the right, the asteroids 1992 SK, 1998 KY26, Castalia, Golevka, Nereus and 1999 KW4 shape models. All of them consist of thousands of triangular facets. 

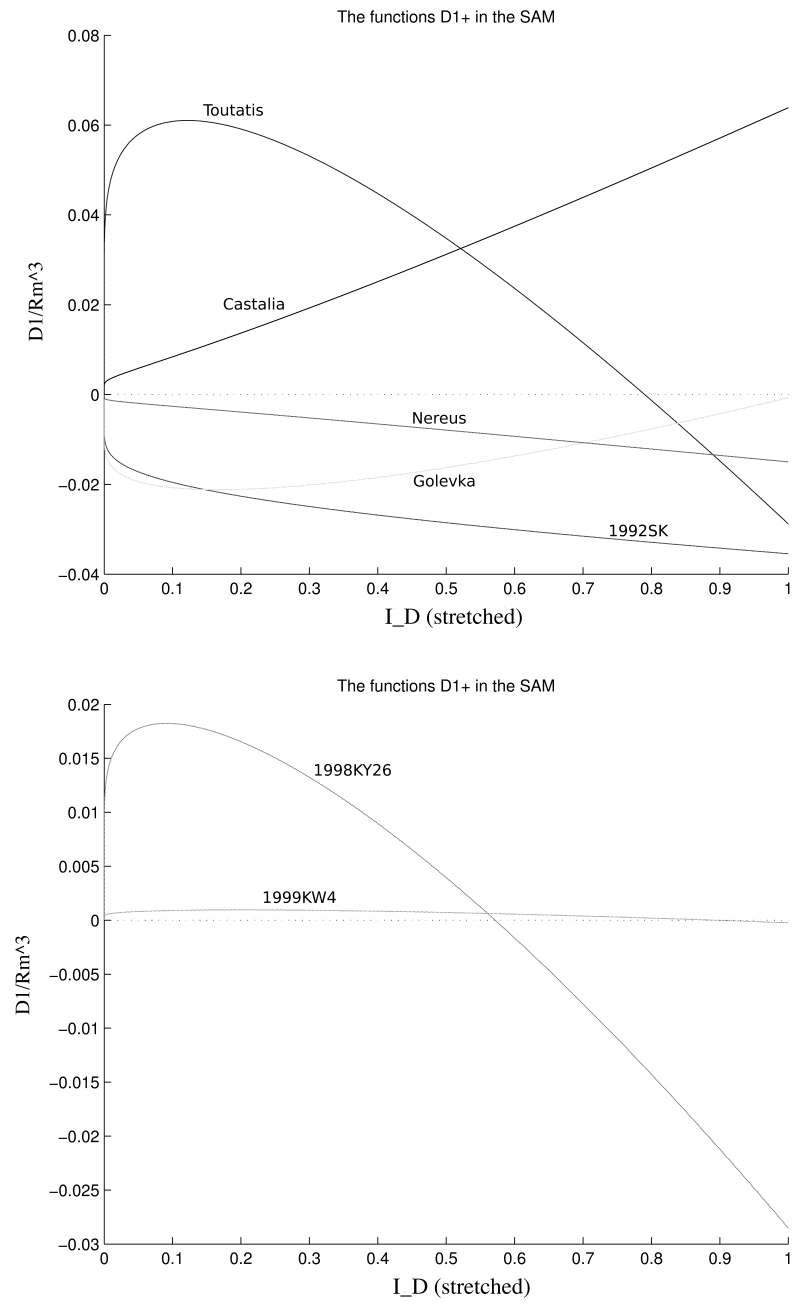

Figure 17: In this plot we show the $\mathcal{D}_{1}$ functions for the seven asteroid shape models we tested, normalized by the mean radius cube $R_{m}^{3}$ of the asteroid, for the $S A M_{+}$case. The horizontal axis is stretched for each asteroid in order to show the plots together $((B, C) \rightarrow(0,1))$. The zero value is highlighted with an horizontal line. 

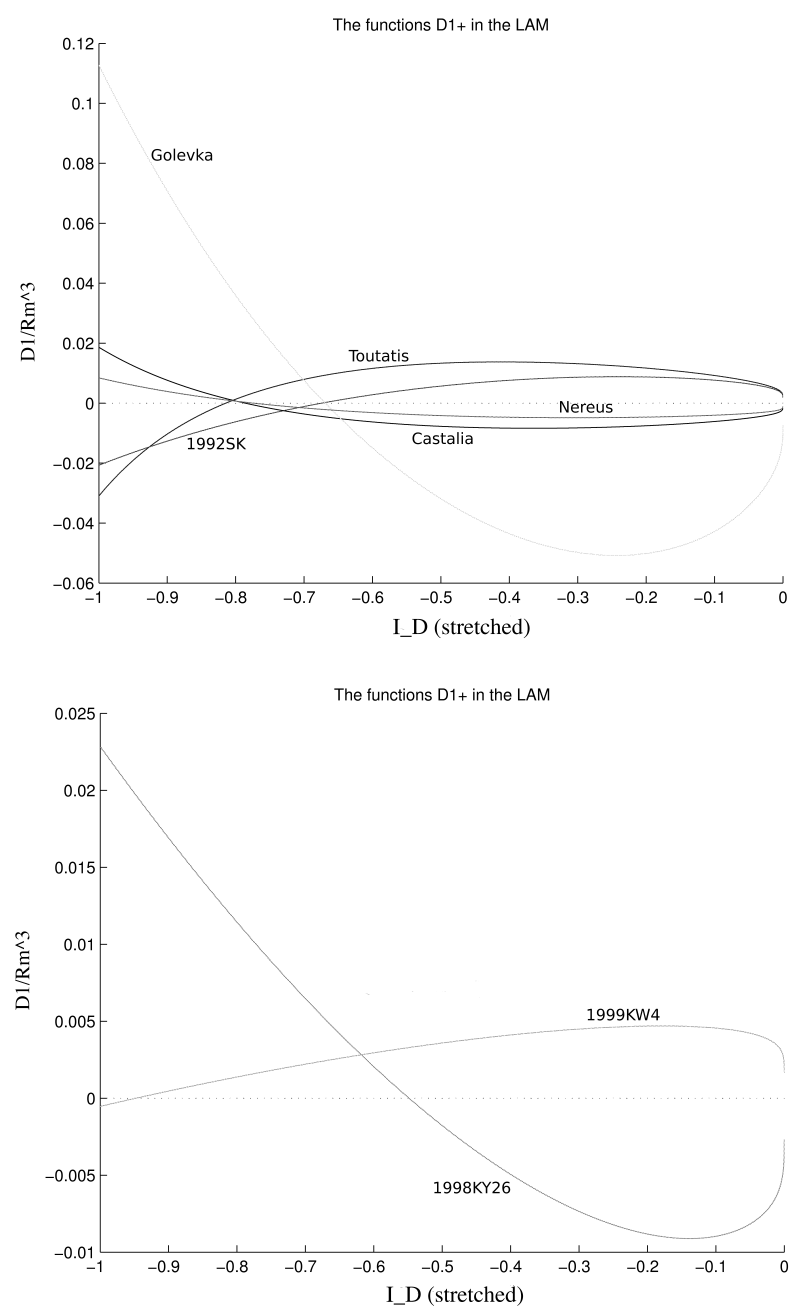

Figure 18: In this plot we show the $\mathcal{D}_{1}$ functions for the seven asteroid shape models we tested, normalized by the mean radius cube $R_{m}^{3}$ of the asteroid, for the $L A M_{+}$case. The horizontal axis is stretched for each asteroid in order to show the plots together $((A, B) \rightarrow(-1,0))$. The zero value is highlighted with an horizontal line. 

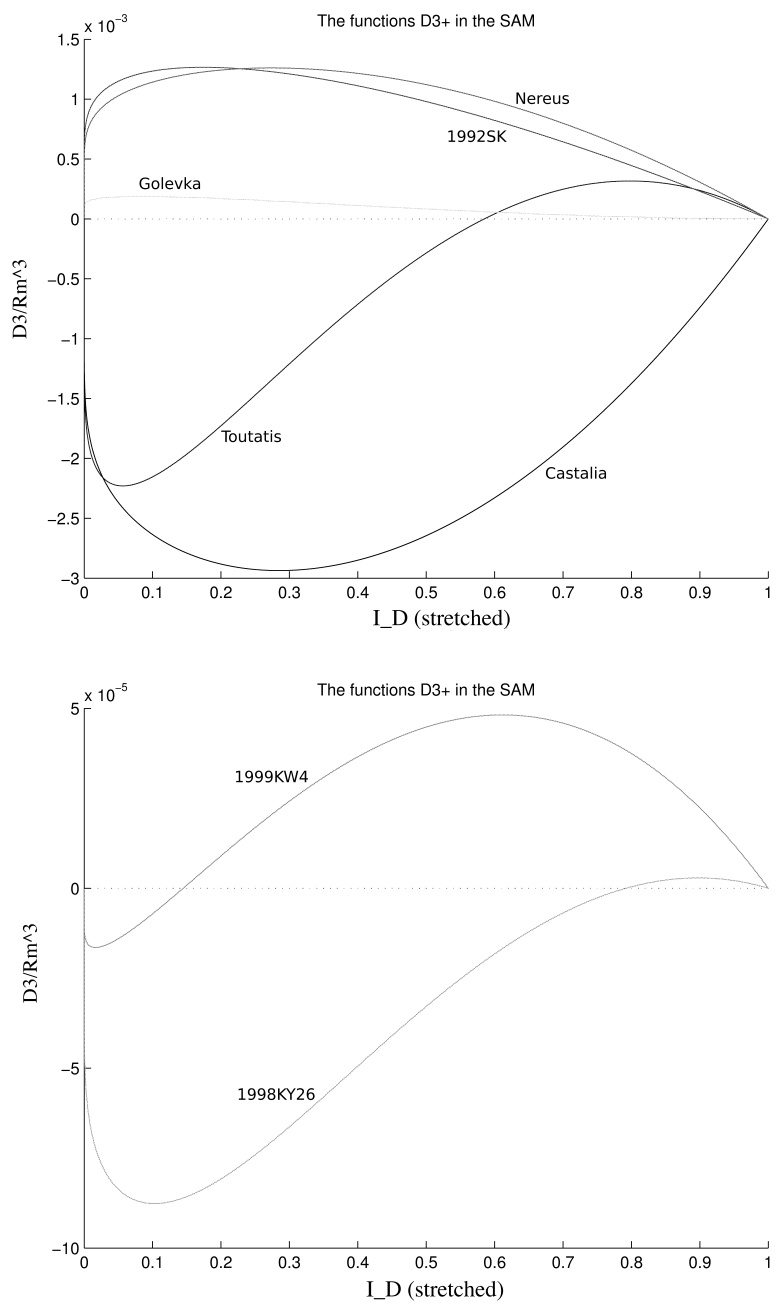

Figure 19: In this plot we show the $\mathcal{D}_{3}$ functions for the seven asteroid shape models we tested, normalized by the mean radius cube $R_{m}^{3}$ of the asteroid, for the $S A M_{+}$case. The horizontal axis is stretched for each asteroid in order to show the plots together $((B, C) \rightarrow(0,1))$. The zero value is highlighted with an horizontal line. 

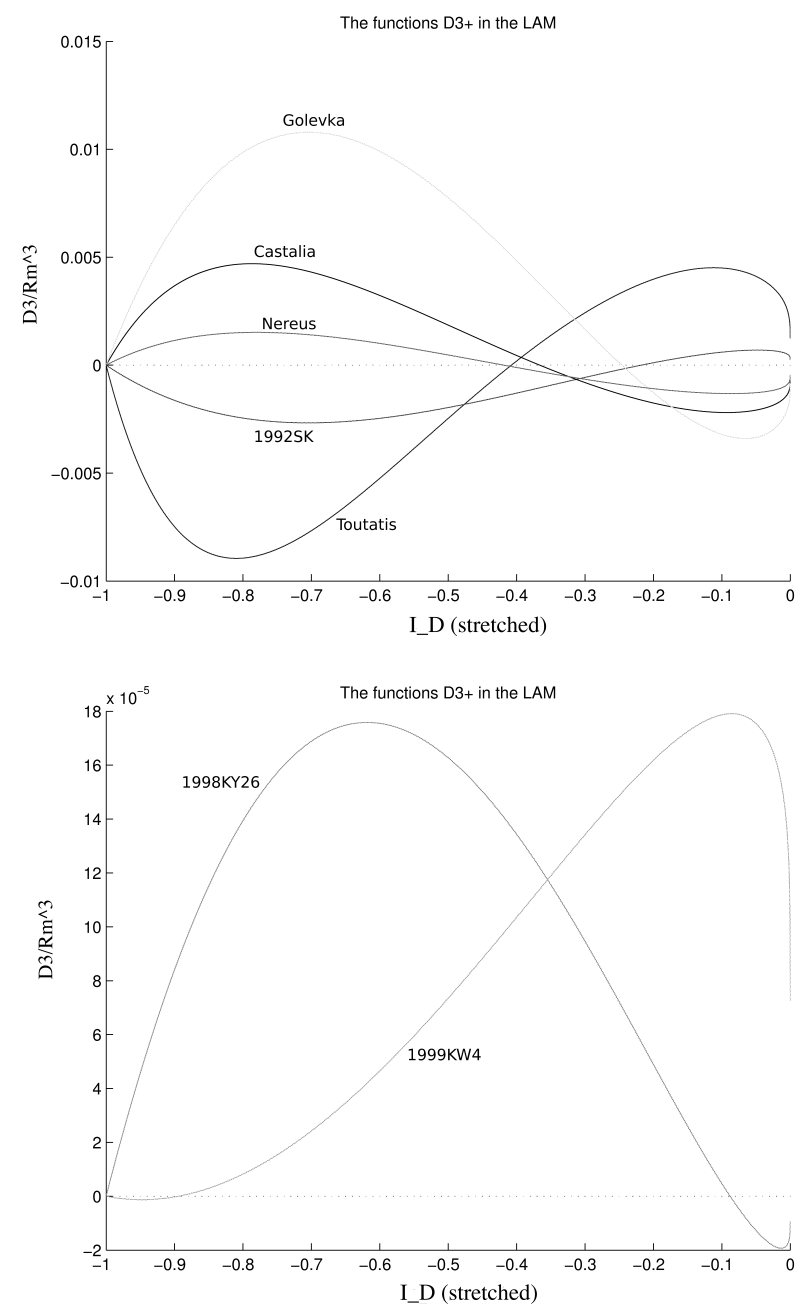

Figure 20: In this plot we show the $\mathcal{D}_{3}$ functions for the seven asteroid shape models we tested, normalized by the mean radius cube $R_{m}^{3}$ of the asteroid, for the $L A M_{+}$case. The horizontal axis is stretched for each asteroid in order to show the plots together $((A, B) \rightarrow(-1,0))$. The zero value is highlighted with an horizontal line. 
We summarize in the next Tables the main features of the $\mathcal{D}_{k}$ functions showed in Figures 17 - 20.

\begin{tabular}{|l|l|l|l|}
\hline & $L A M$ & & \\
\hline Asteroid & \# roots of $\mathcal{D}_{1}$ & \# roots of $\mathcal{D}_{3}$ & Eq. point $\left(\bar{I}_{D}^{e}, \delta^{*}\right)$ \\
\hline Toutatis & 1 & 1 & center \\
\hline 1992 SK & 1 & 1 & center \\
\hline 1998 KY26 & 1 & 1 & center \\
\hline Castalia & 1 & 1 & center \\
\hline Golevka & 1 & 1 & center \\
\hline Nereus & 1 & 1 & center \\
\hline 1999 KW4 & 1 & 1 & center \\
\hline
\end{tabular}

Table 1: The main features of the $\mathcal{D}_{k}$ functions for the asteroid shape models that we tested, for $\bar{I}_{D} \in(A, B)$. The behaviour of the solutions are the ones described in Figure 8.

\begin{tabular}{|l|l|l|l|}
\hline & $S A M$ & & \\
\hline Asteroid & \# roots of $\mathcal{D}_{1}$ & \# roots of $\mathcal{D}_{3}$ & Eq. point $\left(\bar{I}_{D}^{e}, \bar{\delta}^{*}\right)$ \\
\hline Toutatis & 1 & 1 & center \\
\hline 1992 SK & 0 & 0 & - \\
\hline 1998 KY26 & 1 & 1 & center \\
\hline Castalia & 0 & 0 & - \\
\hline Golevka & 0 & 0 & - \\
\hline Nereus & 0 & 0 & - \\
\hline 1999 KW4 & 1 & 1 & center \\
\hline
\end{tabular}

Table 2: The main features of the $\mathcal{D}_{k}$ functions for the asteroid shape models that we tested, for $\bar{I}_{D} \in(B, C)$. The behaviour of the solutions are the ones described in Figures 8 and 9.

We note that in all of the shape models we tested, we only found the behaviours of Figures 8 and 9, and we did not find any of the "hyperbolic" points described in section 4 . We also note that there is no strong preference for the sign of $\mathcal{D}_{k}^{+}$over the different intervals. We note explicitly that the asteroids 1992 SK, Castalia, Golevka and Nereus have a phase plane of the sort found in Figure 8 for the $L A M$ state and of the sort found in Figure 9 for the $S A M$ state. 


\subsection{Tests of the dynamics in detail: Asteroid Toutatis}

In this subsection we perform some numerical simulation using the Toutatis shape model and the rotation state initial conditions given in [Hudson \& Ostro 1995]. As stated in Hudson and Ostro the asteroid Toutatis had the following initial conditions at the epoch 11 Dec 1992:

$$
\theta_{0} \cong 65^{\circ}, \quad \phi_{0} \cong 40^{\circ}, \quad \psi_{0} \cong 134^{\circ}, \quad \rho_{0} \cong 142^{\circ}, \quad \sigma_{0} \cong 136^{\circ},
$$

which means a $L A M_{+}$mode with $I_{D 0} / B \cong 0.54$ and an obliquity of $\delta_{0} \cong 65^{\circ}$. The asteroid's mass is $m \cong 1.5 \times 10^{13} \mathrm{~kg}$, the moments of inertia over mass ratios are $A / m \cong 0.37 \mathrm{~km}^{2}, B / m \cong 1.11 \mathrm{~km}^{2}, C / m \cong 1.18 \mathrm{~km}^{2}$.

The numerical integration of the averaged equations, for a time span of $\Delta t \cong 10^{6} T_{\text {orb }}, T_{\text {orb }} \cong 4$ years, is described in the next Figures 21 and 22 . Note that the solution has to stay in a level curve of the first integral (21), the orbit is closed and it undergoes one full cycle in the plane $\left(\bar{I}_{D}, \bar{\delta}\right)$.
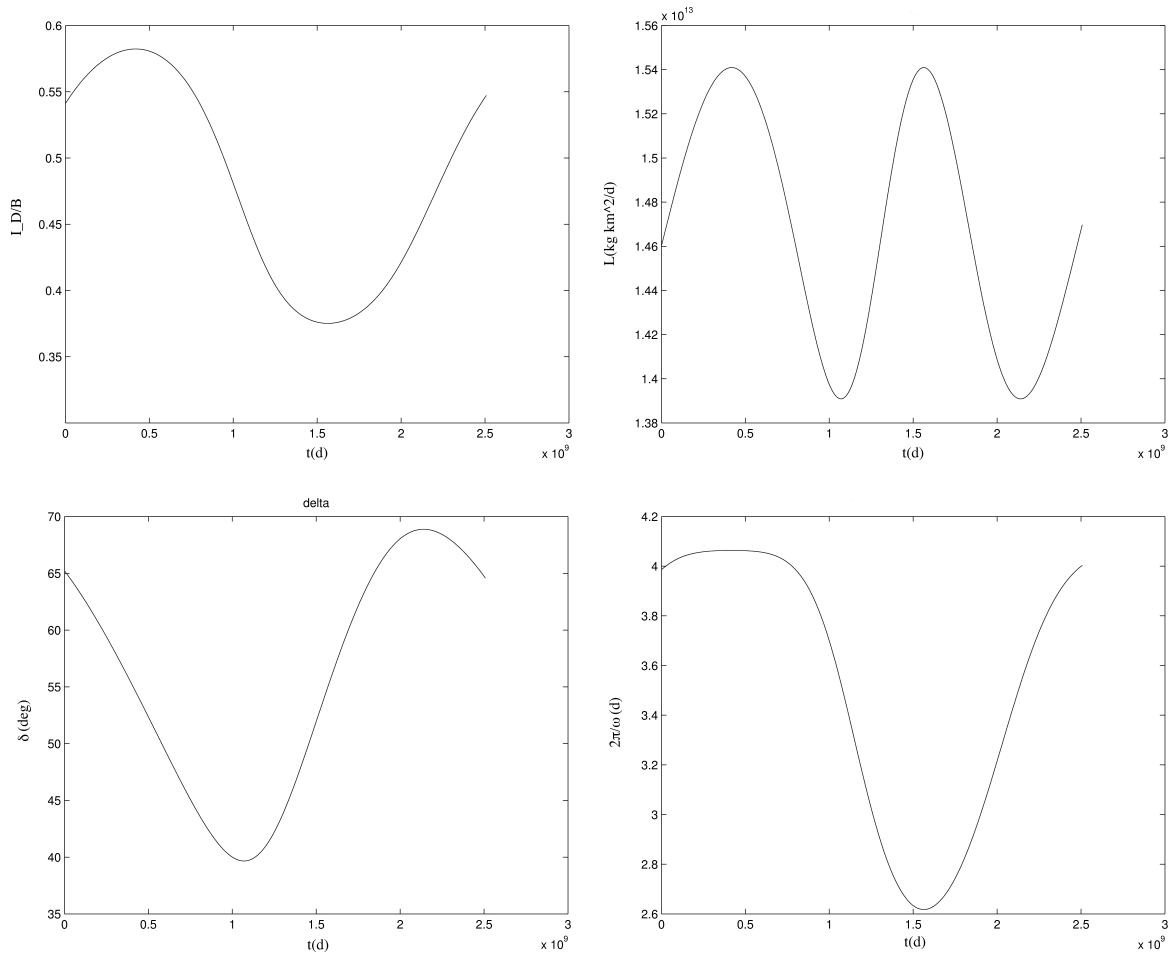

Figure 21: Fromt the top on the left to the bottom on the right: the dynamic inertia $\bar{I}_{D} / B$, the angular momentum magnitude $\bar{L}$, the averaged obliquity $\bar{\delta}$ and the effective rotational period $2 \pi / \bar{\omega}_{l}$ over time, $\bar{\omega}_{l}=\bar{L} / \bar{I}_{D}$. 
As we noticed in section 4.5, if we also add the effect of a small energy dissipation through the formulas (23) and (24), the solution does not remain on the level curve of the first integral of formula (21), and it tends to the equilibrium point (asymptotic value) in the case that stability is introduced, and otherwise it goes away from it, , which is the case that occurs in Figure 22 .

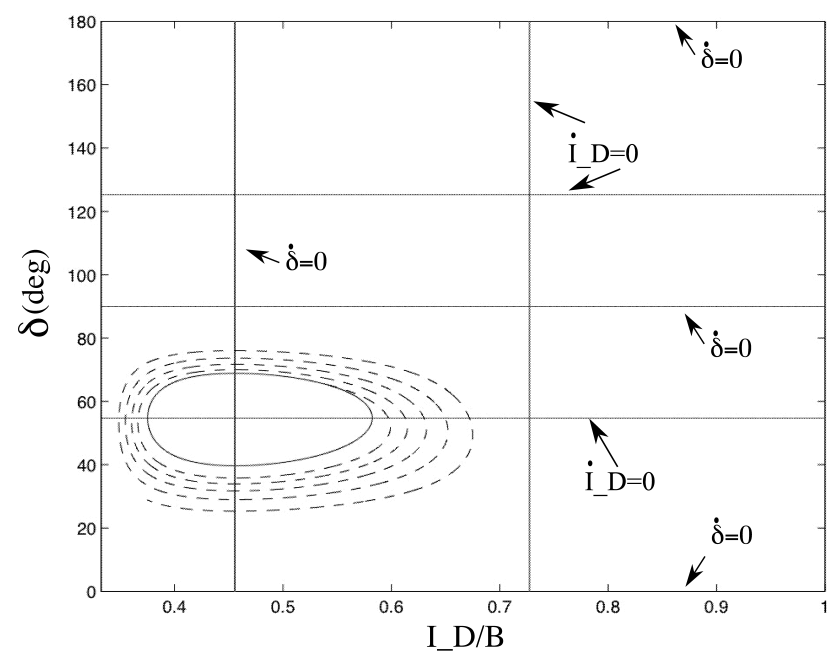

Figure 22: The secular solution (solid line) stays in a closed cycle around the equilibrium point $\left(\bar{I}_{D}^{e}, \sim 55^{\circ}\right)$ on the plane $\left(\bar{I}_{D}, \bar{\delta}\right)$, where $\mathcal{D}_{1}\left(\bar{I}_{D}^{e}\right)=$ $0, \bar{I}_{D}^{e} \in(A, B)\left(L A M_{+}\right)$. The dashed curve is the solution with a small energy dissipation, enhanced enough to be clearly seen $\left(\varrho \cong 2 \mathrm{~g} / \mathrm{cm}^{3}, \mu Q \cong\right.$ $\left.10^{10} \mathrm{~g} / \mathrm{cm} \mathrm{s}^{2}\right)$ and integrated over a long enough time span $\left(\Delta t \cong 10^{7} T_{\text {orb }}\right)$. Its effect is to drive the solution far from the equilibrium point on a spiral. $\bar{I}_{D}$ is scaled by the value $B$, the stationary values for $\bar{I}_{D}$ (vertical lines) and $\bar{\delta}$ (horizontal lines) are highlighted. 


\section{Conclusions}

In this paper we developed the first analytical approach to the problem of a rigid body in non-principal axis rotation under the YORP torque.

The method we used is based on a first order approximation of the general rotational equations of motion with a small torque, averaged over the fast rotational angles in order to capture the secular terms. To make the analytical computation easier, we assumed zero thermal inertia and a second order approximation for the illumination function, considering only "convex-like" bodies, hence no possible self-shadowing. Energy dissipation equations are also derived for eventual incorporation into the model.

We obtained simple integrable secular equations for the angular momentum, the dynamic inertia and the obliquity. We tested them on several real asteroid shape models, in particular on the asteroid Toutatis, which is known to be in a non-principal axis state.

These equations show the same basic properties of previous research on principal axis rotators under YORP, like [Scheeres \& Mirrahimi 2008] and [Nesvorny \& Vokrouhlicky 2007], and have main features in agreement with the numerical simulations of [Vokrouhlicky et al. 2007], although also some non negligible differences as the closed cycles around the equilibrium points which correspond instead to asymptotic states in their numerical simulations.

We believe that the main limit of the current approach is in the model of the illumination function, besides the zero termal lag approximation. As pointed out in [Statler 2009], small changes in the surface of the body, and so in the illumination model, yield significant effects on the rotation.

A better analytical model for the illumination of the body, including selfshadowing and finite thermal inertia, is necessary to describe the rotational dynamics under the YORP torque in a more accurate way. Whether a more detailed analytical approach can be afforded or not, is a challenge for future improvements of this work.

\section{APPENDIX: The averaged factors}

Once we have chosen the initial conditions of the dynamical system, we have a nominal initial value for $I_{D}=I_{D 0}$, which is constant in the unperturbed solution. We distinguish between $S A M$ mode, if $B<I_{D 0}<C$, or $L A M$ mode, if $A<I_{D 0}<B$.

The complete classification of the unperturbed motion is given by the following rule:

$$
\begin{aligned}
& S A M_{+} \Leftrightarrow\left\{B<I_{D}<C \wedge \cos \theta>0\right\}, S A M_{-} \Leftrightarrow\left\{B<I_{D}<C \wedge \cos \theta<0\right\}, \\
& L A M_{+} \Leftrightarrow\left\{A<I_{D}<B \wedge \sin \psi>0\right\}, L A M_{-} \Leftrightarrow\left\{A<I_{D}<B \wedge \cos \psi<0\right\} .
\end{aligned}
$$


In the following, let be

$$
k^{2}=\frac{(B-A)\left(C-I_{D}\right)}{\left(I_{D}-A\right)(C-B)} \quad S A M, \quad k^{2}=\frac{\left(I_{D}-A\right)(C-B)}{(B-A)\left(C-I_{D}\right)} \quad L A M
$$

and $K$ and $E$ are, respectively, the complete elliptic integrals of the first and of the second kind, with parameter $k$. We indicate with \pm the rotation mode $S A M_{ \pm}$or $L A M_{ \pm}$.

The time dependance of the quantities $a_{* *}$ through the unperturbed angles $\theta, \psi$ has always a period $P_{\theta \psi}=4 K$. All the following formulas follow from the properties of the elliptic functions $s n, c n, d n$ with parameter $k$.

$$
\begin{aligned}
& <a_{z \xi}>_{e}=\frac{1}{4 K} \sqrt{\frac{A\left(C-I_{D}\right)}{I_{D}(C-A)}} \int_{0}^{4 K} c n(\tau) d \tau=0 \quad S A M \\
& = \pm \frac{1}{4 K} \sqrt{\frac{A\left(C-I_{D}\right)}{I_{D}(C-A)}} \int_{0}^{4 K} d n(\tau) d \tau= \pm \frac{\pi}{2 K} \sqrt{\frac{A\left(C-I_{D}\right)}{I_{D}(C-A)}} \quad L A M \\
& <a_{z \eta}>_{e}=\frac{1}{4 K} \sqrt{\frac{B\left(C-I_{D}\right)}{I_{D}(C-B)}} \int_{0}^{4 K} \operatorname{sn}(\tau) d \tau=0 \quad S A M \\
& =\frac{1}{4 K} k \sqrt{\frac{B\left(C-I_{D}\right)}{I_{D}(C-B)}} \int_{0}^{4 K} \operatorname{sn}(\tau) d \tau=0 \quad L A M \\
& <a_{z \zeta}>_{e}= \pm \frac{1}{4 K} \sqrt{\frac{C\left(I_{D}-A\right)}{I_{D}(C-A)}} \int_{0}^{4 K} d n(\tau) d \tau= \pm \frac{\pi}{2 K} \sqrt{\frac{C\left(I_{D}-A\right)}{I_{D}(C-A)}} \quad S A M \\
& =\frac{1}{4 K} \sqrt{\frac{C\left(I_{D}-A\right)}{I_{D}(C-A)}} \int_{0}^{4 K} c n(\tau) d \tau=0 \quad L A M .
\end{aligned}
$$

The $z$ - components products:

$$
\begin{aligned}
<a_{z \xi} a_{z \eta}>_{e}=<a_{z \xi} a_{z \zeta}>_{e}=<a_{z \eta} a_{z \zeta}>_{e}=0 \\
<a_{z \xi}^{2}>_{e}=\frac{A\left(C-I_{D}\right)}{I_{D}(C-A)}\left(1-\frac{1}{k^{2}}\left(1-\frac{E}{K}\right)\right) \quad S A M \\
=\frac{A\left(C-I_{D}\right)}{I_{D}(C-A)} \frac{E}{K} L A M \\
<a_{z \eta}^{2}>_{e}=\frac{B\left(I_{D}-A\right)}{I_{D}(B-A)}\left(1-\frac{E}{K}\right) S A M \\
=\frac{B\left(C-I_{D}\right)}{I_{D}(C-B)}\left(1-\frac{E}{K}\right) L A M
\end{aligned}
$$




$$
\begin{aligned}
<a_{z \zeta}^{2}>_{e} & =\frac{C\left(I_{D}-A\right)}{I_{D}(C-A)} \frac{E}{K} \quad S A M \\
& =\frac{C\left(I_{D}-A\right)}{I_{D}(C-A)}\left(1-\frac{1}{k^{2}}\left(1-\frac{E}{K}\right)\right) \text { LAM. }
\end{aligned}
$$

The $z$-components triple products with non zero average in the $S A M$ case are:

$$
\begin{aligned}
& <a_{z \zeta}^{3}>_{e}= \pm\left(\frac{C\left(I_{D}-A\right)}{I_{D}(C-A)}\right)^{3 / 2} \frac{\pi}{4 K}\left(2-k^{2}\right), \\
& <a_{z \xi}^{2} a_{z \zeta}>_{e}= \pm \frac{A\left(C-I_{D}\right)}{I_{D}(C-A)} \sqrt{\frac{C\left(I_{D}-A\right)}{I_{D}(C-A)}} \frac{\pi}{4 K} \\
& <a_{z \eta}^{2} a_{z \zeta}>_{e}= \pm \frac{B\left(C-I_{D}\right)}{I_{D}(C-B)} \sqrt{\frac{C\left(I_{D}-A\right)}{I_{D}(C-A)}} \frac{\pi}{4 K} .
\end{aligned}
$$

The $z$-components triple products with non zero average in the $L A M$ case are:

$$
\begin{gathered}
<a_{z \xi}^{3}>_{e}= \pm\left(\frac{A\left(C-I_{D}\right)}{I_{D}(C-A)}\right)^{3 / 2} \frac{\pi}{4 K}\left(2-k^{2}\right), \\
<a_{z \xi} a_{z \zeta}^{2}>_{e}= \pm \frac{C\left(I_{D}-A\right)}{I_{D}(C-A)} \sqrt{\frac{A\left(C-I_{D}\right)}{I_{D}(C-A)}} \frac{\pi}{4 K} \\
<a_{z \xi} a_{z \eta}^{2}>_{e}= \pm \frac{B\left(C-I_{D}\right)}{I_{D}(C-B)} \sqrt{\frac{A\left(C-I_{D}\right)}{I_{D}(C-A)}} k^{2} \frac{\pi}{4 K} .
\end{gathered}
$$

The other averaged factors that we used in the dissipation torque are the following, in the $S A M_{ \pm}$case:

$$
\begin{gathered}
<a_{z \zeta}^{5}>_{e}= \pm\left(\frac{C\left(I_{D}-A\right)}{I_{D}(C-A)}\right)^{5 / 2} \frac{\pi}{4 K}\left(2-2 k^{2}+\frac{3}{4} k^{4}\right), \\
<a_{z \xi}^{2} a_{z \zeta}^{3}>_{e}= \pm \frac{A\left(C-I_{D}\right)}{I_{D}(C-A)}\left(\frac{C\left(I_{D}-A\right)}{I_{D}(C-A)}\right)^{3 / 2} \frac{\pi}{4 K}\left(1-\frac{k^{2}}{4}\right), \\
<a_{z \eta}^{2} a_{z \zeta}^{3}>_{e}= \pm \frac{B\left(C-I_{D}\right)}{I_{D}(C-B)}\left(\frac{C\left(I_{D}-A\right)}{I_{D}(C-A)}\right)^{3 / 2} \frac{\pi}{4 K}\left(1-\frac{3 k^{2}}{4}\right),
\end{gathered}
$$

and in the $L A M_{ \pm}$case:

$$
\begin{gathered}
<a_{z \xi}^{5}>_{e}= \pm\left(\frac{A\left(C-I_{D}\right)}{I_{D}(C-A)}\right)^{5 / 2} \frac{\pi}{4 K}\left(2-2 k^{2}+\frac{3}{4} k^{4}\right), \\
<a_{z \xi}^{3} a_{z \zeta}^{2}>_{e}= \pm \frac{C\left(I_{D}-A\right)}{I_{D}(C-A)}\left(\frac{A\left(C-I_{D}\right)}{I_{D}(C-A)}\right)^{3 / 2} \frac{\pi}{4 K}\left(1-\frac{k^{2}}{4}\right),
\end{gathered}
$$




$$
<a_{z \xi}^{3} a_{z \eta}^{2}>_{e}= \pm \frac{B\left(C-I_{D}\right)}{I_{D}(C-B)}\left(\frac{A\left(C-I_{D}\right)}{I_{D}(C-A)}\right)^{3 / 2} k^{2} \frac{\pi}{4 K}\left(1-\frac{3 k^{2}}{4}\right) .
$$

\section{Acknowledgements}

The funding for this work was provided by the University of Pisa, "Scuola di Dottorato Galileo Galilei", Department of Mathematics. D.J. Scheeres acknowledges support from a NASA Planetary Geology and Geophysics grant. The authors want to thank D. Vokrouhlicky for his insightful suggestions and S. Breiter who, as a referee, helped to improve a lot the preliminar version of this work.

\section{References}

[Arnold et al. 1998] Arnold, V.I., Kozlov V.V., Neishtadt, A.I.: Mathematical aspects of classical and celestial mechanics Encyclopaedia of Mathematical Science, 3. Springer, Berlin, (1998)

[Brozovic et al. 2009] M. Brozovic, et al. Radar observations and a physical model of Asteroid 4660 Nereus, a prime space mission target. Icarus 201 153-166 (2009).

[Busch et al 2006] Busch, M.W., et al.: Radar and optical observations and physical modeling of near-Earth Asteroid 10115 (1992 SK). Icarus 181, 145-155 (2006).

[Capek \& Vokrouhlicky 2004] Capek, D., Vokrouhlicky, D.: The YORP effect with finite thermal conductivity. Icarus 172, 526-536, (2004).

[Hudson \& Ostro 1995] Hudson, R.S., Ostro, S.J.: Shape and Non-Principal axis spin state of asteroid 4179 Toutatis. Science 270, 84-86, (1995).

[Mysen 2007] Mysen, E.: The dynamics of globally active triaxial comets, with applications to asteroid rotation. Mon.Not.R.Astron.Soc. 381, 301307, (2007)

[Mysen 2008] Mysen, E.: An analytical model for YORP and Yarkovsky effects with a physical thermal lag. A\&A 484, 563-573 (2008)

[Neese 2004] Neese, C., Ed. Small Body Radar Shape Models V2.0. EAR-A5-DDR-RADARSHAPE-MODELS-V2.0. NASA Planetary Data System, (2004).

[Neishtadt et al. 2003] Neishtadt, A.I., Scheeres, D.J., Sidorenko, V.V., Stooke, P.J., Vasilev, A.A.: The influence of reactive torques on comet nucleus rotation. CMDA 86, 249-275, (2003) 
[Nesvorny \& Vokrouhlicky 2007] Nesvorny, D., Vokrouhlicky, D.: Analytical theory of the YORP effect for near spherical objects. Astronom J 134, 1750-1768, (2007)

[Ostro et al 2006] Ostro, S.J., et al. Radar Imaging of Binary Near-Earth Asteroid (66391) 1999 KW4. Science 314: 1276, (2006).

[MacMillan 1936] MacMillan, W.D.: Dynamics of Rigid Bodies. McGrawHill, (1936).

[Rubincam 2000] Rubincam, D.P.: Radiative spin-up and spin-down of small asteroids. Icarus 148, 2-11, (2000).

[Scheeres 2007] Scheeres, D.J.: The dynamical evolution of uniformly rotating asteroids subject to YORP. Icarus 188, 430-450, (2007).

[Scheeres \& Mirrahimi 2008] Scheeres, D.J., Mirrahimi, S.: Rotational dynamics of a solar system body under solar radiation torque. CMDA 101, 69-103, (2008)

[Sharma et al. 2005] Sharma, I., Burns, J.A., Hui, C.Y.: Nutational damping times in solids of revolution. Mon.Not.R.Astron.Soc. 359, 79-92, (2005)

[Sidorenko et al. 2008] Sidorenko V.V., Scheeres D.J., Byram S.M.: On the rotation of comet Borrelly's nucleus. CMDA 102, 133-147, (2008).

[Statler 2009] Statler, T.S.: Extreme Sensitivity of the YORP Effect to Small-Scale Topography. Icarus 202, Issue 2, 502-513, (2009).

[Vokrouhlicky et al. 2007] Vokrouhlicky, D., Breiter, S., Nesvorny, D., Bottke, W.F.: Generalized YORP evolution: onset of tumbling and new asymptotic states. Icarus 191, 636-650, (2007)

[Vokrouhlicky \& Capek 2002] Vokrouhlicky, D., Capek, D.: YORP-induced long term evolution of the spin state of small asteroids and meteoroids: Rubincam's approximation. Icarus 159, 449-467, (2002) 